\title{
First LOFAR observations at very low frequencies of cluster-scale non-thermal emission: the case of Abell 2256
}

\author{
R. J. van Weeren ${ }^{1,2}$, H. J. A. Röttgering ${ }^{1}$, D. A. Rafferty ${ }^{1}$, R. Pizzo ${ }^{2}$, A. Bonafede ${ }^{3}$, M. Brüggen ${ }^{3}$, G. Brunetti ${ }^{4}$ \\ C. Ferrari ${ }^{5}$, E. Orrù ${ }^{6}$, G. Heald ${ }^{2}$, J. P. McKean ${ }^{2}$, C. Tasse ${ }^{7}$, F. de Gasperin ${ }^{8}$, L. Bîrzan ${ }^{1}$, J. E. van Zwieten ${ }^{2}$, \\ S. van der Tol ${ }^{1}$, A. Shulevski ${ }^{9}$, N. Jackson ${ }^{10}$, A. R. Offringa ${ }^{9}$, J. Conway ${ }^{11}$, H. T. Intema ${ }^{12}$, T. E. Clarke ${ }^{13}$,
} I. van Bemmel ${ }^{2}$, G. K. Miley ${ }^{1}$, G. J. White ${ }^{14,15}$, M. Hoeft ${ }^{16}$, R. Cassano ${ }^{4}$, G. Macario ${ }^{5}$, R. Morganti ${ }^{2,9}$, M. W. Wise ${ }^{2,17}$, C. Horellou ${ }^{11}$, E. A. Valentijn ${ }^{8}$, O. Wucknitz ${ }^{18}$, K. Kuijken ${ }^{1}$, T. A. Enßlin ${ }^{8}$, J. Anderson ${ }^{19}$, A. Asgekar ${ }^{2}$, I. M. Avruch ${ }^{2,9}$, R. Beck ${ }^{19}$, M. E. Bell ${ }^{20}$, M. R. Bell ${ }^{8}$, M. J. Bentum ${ }^{2}$, G. Bernardi ${ }^{21}$, P. Best ${ }^{22}$, A.-J. Boonstra ${ }^{2}$, M. Brentjens ${ }^{2}$, R. H. van de Brink ${ }^{2}$, J. Broderick ${ }^{20}$, W. N. Brouw ${ }^{2,9}$, H. R. Butcher ${ }^{2,23}$, W. van Cappellen ${ }^{2}$, B. Ciardi ${ }^{8}$, J. Eislöffel ${ }^{16}$, H. Falcke ${ }^{6,2}$, R. Fender ${ }^{20}$, M. A. Garrett ${ }^{2,1}$, M. Gerbers ${ }^{2}$, A. Gunst ${ }^{2}$, M. P. van Haarlem² ${ }^{2}$, J. P. Hamaker ${ }^{2}$, T. Hassall ${ }^{10}$, J. W. T. Hessels ${ }^{2,17}$, L. V. E. Koopmans ${ }^{9}$, G. Kuper ${ }^{2}$, J. van Leeuwen²,17, P. Maat ${ }^{2}$, R. Millenaar ${ }^{2}$, H. Munk ${ }^{2}$, R. Nijboer ${ }^{2}$, J. E. Noordam ${ }^{2}$, V. N. Pandey ${ }^{9}$, M. Pandey-Pommier ${ }^{1,24}$, A. Polatidis ${ }^{2}$, W. Reich ${ }^{19}$, A. M. M. Scaife ${ }^{20}$, A. Schoenmakers ${ }^{2}$, J. Sluman ${ }^{2}$, B. W. Stappers ${ }^{10}$, M. Steinmetz ${ }^{25}$, J. Swinbank ${ }^{17}$, M. Tagger ${ }^{26}$, Y. Tang ${ }^{2}$, R. Vermeulen ${ }^{2}$, M. de Vos ${ }^{2}$, and M. P. van Haarlem ${ }^{2}$

(Affiliations can be found after the references)

Received 2 March 2012 / Accepted 12 April 2012

\begin{abstract}
Abell 2256 is one of the best known examples of a galaxy cluster hosting large-scale diffuse radio emission that is unrelated to individual galaxies. It contains both a giant radio halo and a relic, as well as a number of head-tail sources and smaller diffuse steep-spectrum radio sources. The origin of radio halos and relics is still being debated, but over the last years it has become clear that the presence of these radio sources is closely related to galaxy cluster merger events. Here we present the results from the first LOFAR low band antenna (LBA) observations of Abell 2256 between 18 and $67 \mathrm{MHz}$. To our knowledge, the image presented in this paper at $63 \mathrm{MHz}$ is the deepest ever obtained at frequencies below $100 \mathrm{MHz}$ in general. Both the radio halo and the giant relic are detected in the image at $63 \mathrm{MHz}$, and the diffuse radio emission remains visible at frequencies as low as $20 \mathrm{MHz}$. The observations confirm the presence of a previously claimed ultra-steep spectrum source to the west of the cluster center with a spectral index of $-2.3 \pm 0.4$ between 63 and $153 \mathrm{MHz}$. The steep spectrum suggests that this source is an old part of a head-tail radio source in the cluster. For the radio relic we find an integrated spectral index of $-0.81 \pm 0.03$, after removing the flux contribution from the other sources. This is relatively flat which could indicate that the efficiency of particle acceleration at the shock substantially changed in the last $\sim 0.1$ Gyr due to an increase of the shock Mach number. In an alternative scenario, particles are re-accelerated by some mechanism in the downstream region of the shock, resulting in the relatively flat integrated radio spectrum. In the radio halo region we find indications of low-frequency spectral steepening which may suggest that relativistic particles are accelerated in a rather inhomogeneous turbulent region.
\end{abstract}

Key words. telescopes - radio continuum: general - galaxies: clusters: individual: Abell 2256 - large-scale structure of Universe

\section{Introduction}

Radio halos and relics are diffuse radio sources, unrelated to individual galaxies, found in some disturbed galaxy clusters (see the reviews by Feretti 2005; Ferrari et al. 2008; Venturi 2011, and references therein). These sources are not ubiquitous in galaxy clusters - only a few dozen examples have been previously identified. The presence of diffuse radio emission indicates magnetic fields and relativistic particles in the intracluster medium (ICM). Due to their limited lifetime, the synchrotron emitting electrons need to be (re)accelerated in situ (Jaffe 1977). The origin of this diffuse emission is presently still being debated and is crucial for understanding the non-thermal component in the ICM and particle acceleration mechanisms.

It is predicted in the framework of the concordant cosmological model that galaxy clusters grow as a result of the mergers of smaller clusters and sub-structures, which create turbulence and shocks in their ICM (e.g., Markevitch et al. 2002; Vazza et al. 2006; Russell et al. 2010). Over the last decade it has become clear that the diffuse radio emission in galaxy clusters is often related to galaxy cluster merger events (e.g., Cassano et al. 2010b; van Weeren et al. 2010).

Diffuse sources in merging clusters have commonly been classified as radio relics and halos. In this context, elongated filamentary sources have generally been called relics. Relics are usually further subdivided into three classes (see also Kempner et al. 2004). (1) Radio Gischt are large extended arc-like sources mostly found in the outskirts of galaxy clusters. They are often highly polarized, $20 \%$ or more, at frequencies $\gtrsim 1 \mathrm{GHz}$. It has been proposed that they directly trace shock waves (Enßlin et al. 1998; Miniati et al. 2001), in which particles are accelerated by the diffusive shock acceleration mechanism in a firstorder Fermi process (DSA; Blandford \& Ostriker 1978; Drury 1983; Blandford \& Eichler 1987; Jones \& Ellison 1991; Malkov \& O'C Drury 2001). A related scenario is that of shock reacceleration of pre-accelerated electrons in the ICM. This may be a more efficient mechanism in weak shocks (e.g., Markevitch et al. 2005; Giacintucci et al. 2008; Kang \& Ryu 2011) and might 
be needed because the efficiency with which collisionless shocks can accelerate particles is unknown, and might not be enough to produce the observed radio brightness (e.g., Macario et al. 2011). The pre-accelerated electrons could for example originate from the radio galaxies in clusters. (2) Radio phoenices are most likely related to fossil radio plasma from previous episodes of AGN activity adiabatically compressed by merger shock waves, boosting the radio emission (Enßlin \& Gopal-Krishna 2001; Enßlin \& Brüggen 2002). (3) AGN relics trace uncompressed radio plasma from previous episodes of AGN activity. The radio spectra of both radio phoenices and AGN relics are expected to be steep ${ }^{1}$ and curved due to synchrotron and inverse Compton (IC) losses.

Radio halos are extended sources with sizes of about a Mpc. They are usually unpolarized, with limits at the several percent level, and the radio emission roughly follows the thermal X-rays from the ICM, implying that the emitting relativistic plasma is co-spatial with the thermal gas in galaxy clusters. Potentially several mechanisms may be responsible for the generation of Mpc scale synchrotron emission from galaxy clusters. Relativistic electrons in the ICM can be re-accelerated in situ through interaction with turbulence generated in the ICM by cluster-cluster mergers (Brunetti et al. 2001; Petrosian 2001), or secondary electrons can be continuously injected in the ICM by inelastic collisions between relativistic and thermal protons (Dennison 1980; Blasi \& Colafrancesco 1999; Dolag \& Enßlin 2000; Enßlin et al. 2011). Models considering a combination of the two mechanisms, namely the re-acceleration of both relativistic protons and their secondaries by magnetohydrodynamical turbulence generated in the ICM have been recently considered (Brunetti \& Blasi 2005; Dolag et al. 2008; Brunetti \& Lazarian 2011).

In the case of several giant radio halos, a pure secondary origin is disfavored from their spectra and/or large extension (e.g., Brunetti et al. 2008; Dallacasa et al. 2009; Donnert et al. 2010; Brown \& Rudnick 2011). In particular, an unrealistic amount of energy in the form of relativistic protons would be required by secondary models to explain radio halos with very steep spectra (Brunetti 2004; Pfrommer \& Enßlin 2004; Brunetti et al. 2008; Macario et al. 2010; van Weeren et al. 2011a). On the other hand, if turbulence plays an important role for the origin of these giant sources, a large number of steep spectrum radio halos is expected to glow up at low radio frequencies (Cassano et al. 2006; Brunetti et al. 2008). The origin of the turbulent magnetic field remains unclear. One possibility is that it has been amplified by dynamo processes acting in the cluster volume (e.g., Ruzmaikin et al. 1989; Dolag et al. 2005, and references therein).

The LOw Frequency ARray (LOFAR) is a new generation radio telescope operating at $10-240 \mathrm{MHz}$ (van Haarlem et al., in prep.). The large collecting area and instantaneous fractional bandwidth, together with long baselines, allow the first detailed study of low-surface brightness diffuse cluster radio sources below $100 \mathrm{MHz}$. In this respect LOFAR is the ideal radio telescope to unveil the existence of steep spectrum diffuse radio emission from galaxy clusters and is expected to provide a breakthrough in the field (Cassano et al. 2010a).

Abell 2256 is a nearby, $z=0.0581$ (Struble \& Rood 1999), cluster that contains a giant $1.2 \mathrm{Mpc}$ radio halo, a relic, and a number of tailed radio galaxies (Bridle \& Fomalont 1976; Bridle et al. 1979; Röttgering et al. 1994; Miller et al. 2003; Intema 2009; van Weeren et al. 2009; Kale \& Dwarakanath 2010). The relic has a large integrated flux, compared to other relics, of about $0.5 \mathrm{Jy}$ at $1.4 \mathrm{GHz}$, and a size of $1125 \mathrm{kpc} \times 520 \mathrm{kpc}$.

\footnotetext{
${ }^{1} \alpha \lesssim-1.5, F_{v} \propto v^{\alpha}$, where $\alpha$ is the spectral index.
}

The average polarization fraction of the relic is about $20 \%$ at $1.4 \mathrm{GHz}$. Clarke \& Enßlin (2006). A spectral analysis by Brentjens (2008) shows that the radio halo component dominates the integrated cluster radio spectrum at very low frequencies. X-ray and optical observations provide strong evidence that A2256 is undergoing a merger event between a main cluster $\left(T_{\text {ICM }} \sim 7-8 \mathrm{keV}\right)$, a major sub-structure $\left(T_{\mathrm{ICM}} \sim 4.5 \mathrm{keV}\right)$ and, possibly, a third infalling group (Briel et al. 1991; Briel \& Henry 1994; Sun et al. 2002; Berrington et al. 2002; Miller et al. 2003). The cluster has an X-ray luminosity of $L_{\mathrm{X}}, 0.1-2.4 \mathrm{keV}=$ $3.7 \times 10^{44} \mathrm{erg} \mathrm{s}^{-1}$ (Ebeling et al. 1998).

The large angular extent of the diffuse emission and its large integrated flux make A2256 a prime target for low-frequency observations which typically suffer from low spatial resolution and sensitivity, compared to observations at high frequencies. At wavelengths larger than $1 \mathrm{~m}$, the diffuse radio emission in A2256 has been studied with the GMRT at $150 \mathrm{MHz}$ (Intema 2009; Kale \& Dwarakanath 2010), and with the WSRT between 115 and $165 \mathrm{MHz}$ (van Weeren et al. 2009; Intema 2009). The VLSS $74 \mathrm{MHz}$ image (Cohen et al. 2007) only shows a hint of diffuse emission, and the $38 \mathrm{MHz} 8 \mathrm{C}$ and $151 \mathrm{MHz} 6 \mathrm{C}$ survey images (Hales et al. 1995; Masson \& Mayer 1978) have too low resolution to properly separate the flux contributions from the discrete sources. The observations by Intema (2009) and van Weeren et al. (2009) showed the presence of three previously unknown diffuse sources with steep radio spectra $(\alpha \lesssim$ $-1.5)$. These steep radio spectra suggest that these sources trace old radio plasma from previous episodes of AGN activity in the cluster.

In this paper we present the first LOFAR observations of Abell 2256, focussing on the frequency range around $63 \mathrm{MHz}$ and the total intensity (stokes $I$ ) images. The layout of this paper is as follows: in Sect. 2 we give an overview of the observations and the data reduction. In Sect. 3 we present the results, including the radio images and a spectral index map. We end with a discussion and the conclusions in Sects. 4 and 5, respectively. Throughout this paper we assume a $\Lambda$ CDM cosmology with $H_{0}=71 \mathrm{~km} \mathrm{~s}^{-1} \mathrm{Mpc}^{-1}, \Omega_{\mathrm{m}}=0.3$, and $\Omega_{\Lambda}=0.7$.

\section{Observations and data reduction}

\subsection{Observations}

Abell 2256 was observed with the LOFAR LBA system on July 15 and 16, 2011, from 18:00 to 04:00 CEST. The observations covered the 18 to $67 \mathrm{MHz}$ frequency range, except for a gap at $40-42 \mathrm{MHz}$ due to strong radio frequency interference (RFI). All four linear correlation products were recorded. The observed frequency range was divided into 244 sub-bands, each having a bandwidth of $0.1953 \mathrm{MHz}$. Each sub-band was subdivided into 64 frequency channels. The integration time was $1 \mathrm{~s}$. The socalled LBA_OUTER configuration was used for the LBA stations, where LBA antenna refers to a dipole pair and station refers to the collection of dipole pairs grouped as one. In the LBA_OUTER configuration 48 (of 96) LBA antennas are used, located mostly in the outer part of the stations (which have diameters of $87 \mathrm{~m}$ ). This increases the sidelobe levels for the station beams but reduces the field of view (FOV) with respect to other station antenna configurations.

We used eight remote and seventeen core stations for the A2256 observation. The baselines are between $90 \mathrm{~m}$ and $80 \mathrm{~km}$ long. The uv-coverage is shown in Fig. 1. The FOV (full width half maximum (FWHM) of the primary beam) is about $4.6^{\circ}$ and $9.2^{\circ}$ at 60 and $30 \mathrm{MHz}$, respectively. It should be noted 

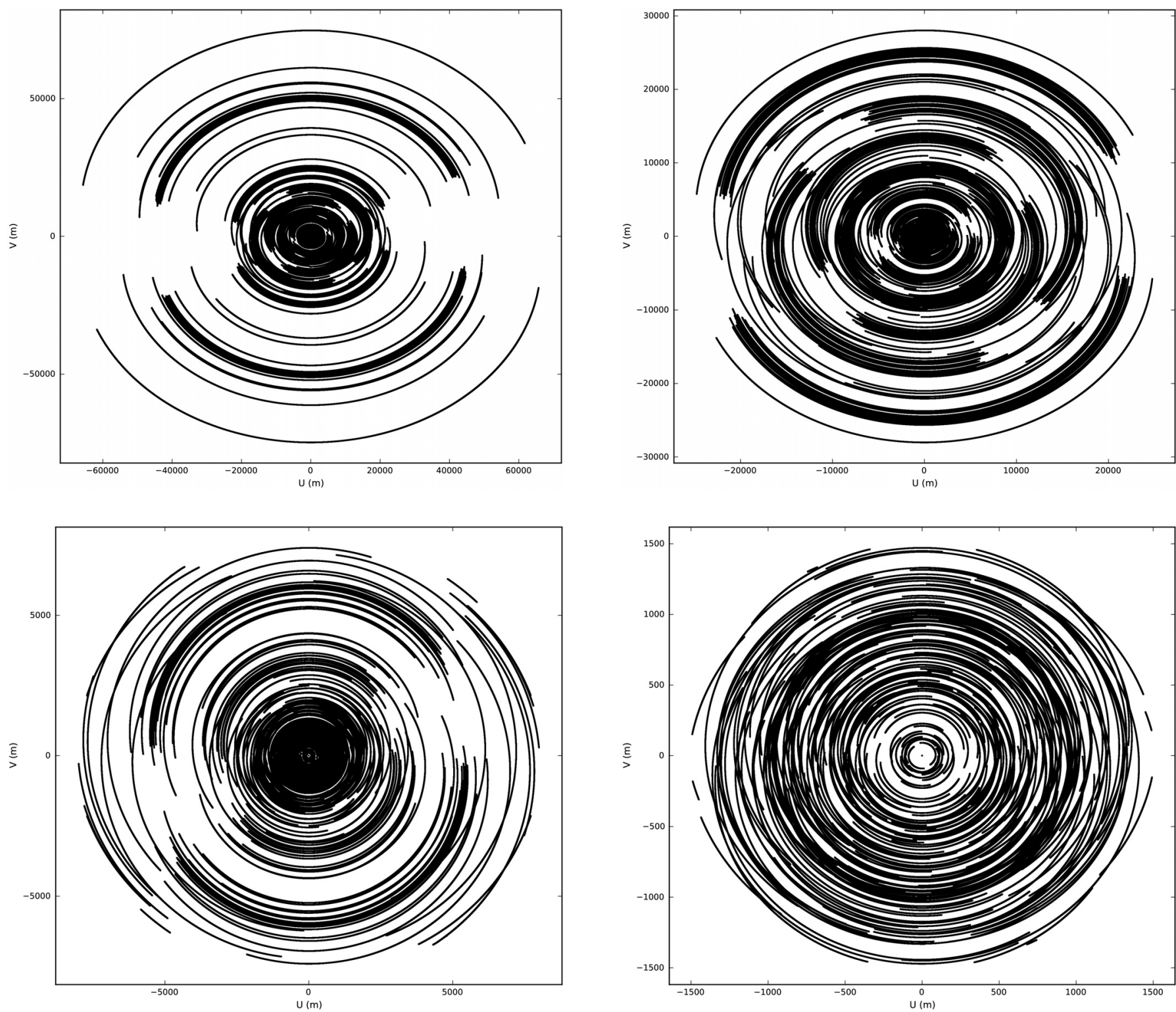

Fig. 1. UV-coverage of the A2256 observation on July 15 and 16, 2011, from 18:00 to 04:00 CEST (10 h in total). Transit occurred around 23:00 CEST. The outer UV-coverage is shown in the top left frame, the next frames progressively zoom inwards. The relatively broad bandwidth fills the uv-plane radially (not shown in the figures).

though that the station beams are complex-valued, time and direction dependent, and differ slightly from station to station.

As a first step, we flagged RFI with the AOFlagger (Offringa et al. 2010) using default settings. The first and last two channels at the edge of each sub-band were removed. The flagger typically found up to a few percent of RFI above $30 \mathrm{MHz}$. The amount of RFI increases strongly below $\sim 28 \mathrm{MHz}$. Between $30 \%$ and $70 \%$ had to be flagged below this frequency for most sub-bands, with at least some of this attributable to short-wave radio transmissions. The RFI situation was somewhat better after midnight. After flagging, we averaged the data in time to $5 \mathrm{~s}$, see Heald et al. (2010) for a brief overview of the data processing steps.

\subsection{Calibration}

For this A2256 observation, the bright "A-team" radio sources Cas A, Cyg A and Vir A are located $34^{\circ}, 42^{\circ}$, and $74^{\circ}$ away from the phase center, respectively. These sources have integrated flux densities of about 18, 17, and $2.1 \mathrm{kJy}$ at $74 \mathrm{MHz}$, respectively. Even after attenuation by the primary beam, Cas A and Cyg A outshine the brightest sources in the target field by more than a factor ten. Their amplitudes are strongly modulated as they move in and out of the station beam sidelobes. For the first $2.5 \mathrm{~h}$ of the observations Vir A also affects the observed visibilities, until the source sets. At frequencies $\lesssim 35 \mathrm{MHz}$, 3C 390.3 (located $4.7^{\circ}$ from the phase center) is sufficiently bright, $145 \pm 14 \mathrm{Jy}$ at $38 \mathrm{MHz}$ (Hales et al. 1995), that it needs to be treated separately as described below.

The first calibration step consisted of the removal of the three "A-team" sources. Below $35 \mathrm{MHz}, 3 \mathrm{C} 390.3$ was also included in the calibration model. For the models of the A-team sources and 3C 390.3 we used the clean component models at $74 \mathrm{MHz}$ from VLA A-array ${ }^{2}$ observations (Kassim et al. 2007) with a resolution of $25^{\prime \prime}$. For all the calibration steps we used the BlackBoard Selfcal (BBS) software system (Pandey et al. 2009).

${ }^{2}$ http://lwa.nrl.navy.mil/tutorial/ 
A\&A 543, A43 (2012)

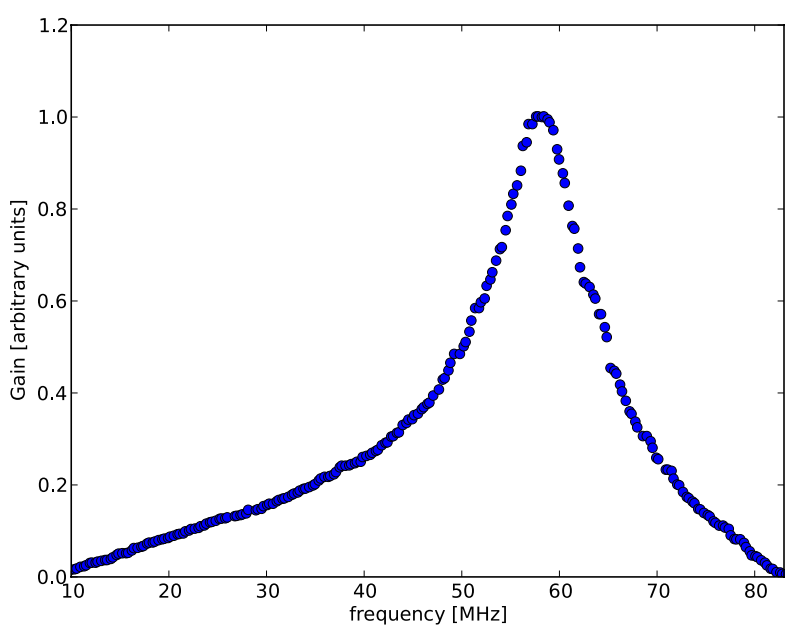

Fig. 2. LBA global bandpass. The bandpass peaks at about $58 \mathrm{MHz}$ as expected due to resonance.

Full polarization direction dependent complex gain solutions were obtained for the three A-team sources (and 3C 390.3 below $35 \mathrm{MHz}$ ) independently per sub-band. Vir A was only included in the calibration model for the first $2.5 \mathrm{~h}$. In general, we obtained high-quality gain solutions for Cas A and Cyg A, while the solutions for Vir A and 3C 390.3 were noisier due to their lower apparent fluxes. The A-team sources were then subtracted (like "peeling") using their direction dependent gain solutions from the visibility data. 3C390.3 was not subtracted because it is in the main FOV below $35 \mathrm{MHz}$ and used in subsequent calibration steps.

After subtraction of the A-team sources from the visibility data we performed another round of flagging with the AOFlagger and averaged the data to 4 channels and $10 \mathrm{~s}$ per sub-band, to decrease the size of the dataset whilst limiting bandwidth and time smearing in the field of view to acceptable levels.

The responses of the LBA antennas depend on the observed frequency, see Fig. 2. The LBA response was obtained from observations of Cyg A. Observations that have been carried out over the course of several months show that the bandpass response is stable at the level of a few percent or better. We divided out this sensitivity pattern to avoid the need to obtain amplitude calibration solutions for each individual sub-band. The reason behind this is that the signal-to-noise ratio (SNR) per sub-band is not sufficient to obtain good gain solutions due to the limited effective bandwidth of $0.183 \mathrm{MHz}$. By combining several subbands, we can overcome this limitation. However, a problem at low frequencies is that the ionospheric phase distortions are frequency dependent, increasing towards lower frequencies. At about $60 \mathrm{MHz}$ this means that if this effect is not included only about $1 \mathrm{MHz}$ bandwidth can be used for calibration, depending on the ionospheric conditions (e.g., Eq. (4) from Intema et al. 2009). To obtain high-quality solutions more bandwidth is required. This can be accomplished by solving for the differential total electron content (TEC). This adds a frequency dependent phase term $(\mathrm{TEC} / v)$. During the calibration we therefore solve for (i) a single overall polarization dependent complex gain factor to set the amplitudes and capture other instrumental effects such as clock drift; and (ii) a polarization independent TEC value per station and time-slot (10 s). We used 20 sub-bands around $20 \mathrm{MHz}, 20$ around $30 \mathrm{MHz}$, and 30 around $63 \mathrm{MHz}$ per solution interval for this "global calibration". Due to computational limitations, we concentrated our efforts on these three frequency ranges, using in total only 70 of the 244 subbands observed.

We calibrated the data against a $74 \mathrm{MHz} 80^{\prime \prime}$ VLSS model of the field around A2256, covering $15^{\circ} \times 15^{\circ}$, and assumed that all sources are unpolarized. This is a reasonable assumption at these low frequencies. We used an overall flux scaling with a spectral index of -0.8 to get a first order approximation of the flux-scale. For computing the model visibilities, we included the complex beam attenuation of each station beam. The station beam model is derived using the dipole beam model based on interpolation of electromagnetic simulations of LBA dipole beam response. The interpolation scheme is described by Hamaker (2011). The beam model does not take into account mutual coupling effects. It is also been assumed that all the dipole pairs/antennas have identical beams.

\subsection{Primary beam correction, flux-scale, and self-calibration}

We applied the calibration solutions and corrected the data for each station's beam response in the phase center. Note that it is only possible to correct for a stations beam response in a single direction. For the other directions within the main FOV this correction is only a first order approximation. The entire FOV, to an attenuation factor of 0.15 compared to the beam center, was then imaged and cleaned using Casapy with w-projection (Cornwell et al. 2005, 2008); $768 \mathrm{w}$-planes were used in total. Ideally, to create flux corrected wide-field images, the time-variable direction dependent effects need to be taken into account (e.g., Bhatnagar et al. 2008). This functionality is still under development for LOFAR data. We made images with "briggs" weighting (Briggs 1995) with robust $=-0.1$. This gives a good resolution of around $25^{\prime \prime}$ at $63 \mathrm{MHz}$, while it still reveals some of the diffuse emission. Using lower values of the robust parameter is not very useful for this dataset because the effective resolution is limited by the ionosphere (as explained later in this subsection). We combined groups of sub-bands around 63, 30 and $20 \mathrm{MHz}$ for multi-frequency synthesis imaging, producing three broadband images.

We simulated a dense grid of 1 Jy point sources in the A2256 field, including the LOFAR station beam, to obtain the correct relative flux scale over the field. This grid is illustrated in Fig. 4. We imaged this simulated dataset (with the same frequency setup and uv-coverage as the observed data) in the same way as the observed A2256 data. We extracted the integrated fluxes for the simulated point sources using the PyBDSM source detection software $^{3}$. A 2D surface was fitted to these extracted source fluxes using the griddata module from the python matplotlib, which employs a Delaunay triangulation ${ }^{4}$. This triangulated surface gives the effective sensitivity as a function of position in the A2256 field and can be used to create a primary beam corrected image. This procedure for correcting a uniform flux scale within the primary beam is a useful stop-gap solution until an a truly beam-aware imager is available. We used the flux-corrected image to obtain an updated sky model (again with PyBDSM) for two subsequent rounds of self-calibration (the calibration strategy remaining unchanged). An example of the phase self-calibration solutions obtained is given in Fig. 5.

\footnotetext{
${ }^{3}$ See the LOFAR Imaging Cookbook at http://www . astron.nl/radio-observatory/lofar/ lofar-imaging-cookbook and http://www.strw.leidenuniv. $\mathrm{nl} /$ mohan/anaamikamanual.pdf

${ }^{4}$ http://matplotlib. sourceforge. net
} 
Table 1. LOFAR LBA image characteristics.

\begin{tabular}{lccc}
\hline \hline $\begin{array}{l}\text { Frequency } \\
\mathrm{MHz}\end{array}$ & $\begin{array}{c}\text { Bandwidth } \\
\mathrm{MHz}\end{array}$ & $\begin{array}{c}\text { Obtained rms } \\
\mathrm{mJy} \mathrm{beam}^{-1}\end{array}$ & $\begin{array}{c}\text { Synthesized beam } \\
\text { arcsec }\end{array}$ \\
\hline 63 (robust -0.1$)$ & 5.5 & 10 & $22 \times 26$ \\
63 (robust 0.5$)$ & 5.5 & 25 & $52 \times 62$ \\
30 (robust -0.1$)$ & 3.7 & 43 & $58 \times 69$ \\
20 (robust -0.1$)$ & 3.7 & 250 & $108 \times 116$ \\
\hline
\end{tabular}

The final images (robust $=-0.1$ ) have noise levels of 10,43 , and $250 \mathrm{mJy} \mathrm{beam}^{-1}$ at 60,30 , and $20 \mathrm{MHz}$, respectively. The noise level at $63 \mathrm{MHz}$ is about a factor of two lower than the deepest VLA $74 \mathrm{MHz}$ images from Lane et al. (2005). A lower resolution (robust $=0.5$ weighting) image at $63 \mathrm{MHz}$ has a noise of $25 \mathrm{mJy}$ beam $^{-1}$, see Table 1 for a summary of the resolution, bandwidth and sensitivity of the images. The thermal noise is about $2.5 \mathrm{mJy}_{\text {beam }}{ }^{-1}$ at $63 \mathrm{MHz}$ and $8 \mathrm{mJy} \mathrm{beam}^{-1}$ at $30 \mathrm{MHz}$. Ionospheric phase errors likely dominate the error budget, especially at 30 and $20 \mathrm{MHz}$. From the simulated point source grid, we estimate that errors coming from the usage of an imager that is unaware of the time-varying LOFAR beam, contribute about $2 \mathrm{mJy}$ beam $^{-1}$ to the noise at $63 \mathrm{MHz}$ in the central part of the FOV. At the time of the A2256 observation the station calibration tables, which contain gain correction factors for the individual dipoles, were still in the process of being refined. This could have resulted in a somewhat reduced station sensitivity.

The presence of residual ionospheric phase errors after calibration lead to a wider point spread function (PSF). We measure a FWHM of the PSF that is about 20" larger than the synthesized beamwidth at $63 \mathrm{MHz}$ in the central part of the FOV. This effect has also been seen for $74 \mathrm{MHz}$ VLA observations (e.g., Cohen et al. 2007). The increase in the PSF FWHM varies between $20^{\prime \prime}$ and $40^{\prime \prime}$ across the FOV, according to the PyBDSM PSF characterization module. We also compared the positions of the brightest sources in the FOV against the catalogue positions from NVSS and WENSS. We find the positional accuracies to be $5^{\prime \prime}$ or better, depending on the SNR and location of the sources. At lower frequencies, the PSF FWHM is about $70^{\prime \prime}$ and $180^{\prime \prime}$ larger (in the central part of the FOV) than the synthesized beamwidth at 30 and $20 \mathrm{MHz}$, respectively. In this case the unresolved sources are elongated, distorted, and partly broken up into smaller components. For this reason, we only use the $63 \mathrm{MHz}$ maps for quantitative analysis in this paper, while the 30 and $20 \mathrm{MHz}$ maps are used for qualitative analysis until a more complete ionospheric correction is made.

To obtain and check the overall flux scale we measured the integrated fluxes for five bright sources in the FOV: 4C +79.16 , $8 \mathrm{C} 1654+785,8 \mathrm{C} 1657+780,8 \mathrm{C} 1708+779$, and 8C 1728+783 (see Fig. 4). For these sources we collected flux density measurements from the $1.4 \mathrm{GHz}$ NVSS (Condon et al. 1998), $325 \mathrm{MHz}$ WENSS (Rengelink et al. 1997), $151 \mathrm{MHz} 7 \mathrm{C}$ (Hales et al. 2007), $74 \mathrm{MHz}$ VLSS (Cohen et al. 2007) and $38 \mathrm{MHz} 8 \mathrm{C}$ (Hales et al. 1995) surveys. We fitted second order polynomials to these flux density measurements in $\log (S)-\log (v)$ space and compared the LOFAR flux density measurements at $63 \mathrm{MHz}$ against the predicted fluxes from the polynomial fits. The median of the correction factors was used to tie the LOFAR images to the flux-scale from these surveys. The correction factor we found was modest, being 1.23 at $63 \mathrm{MHz}$. The spread in the individual correction factors is about 7\%, see Fig. 3.

From this we adopt an error in the relative LOFAR flux scale within the primary beam that is uncertain by $7-10 \%$.

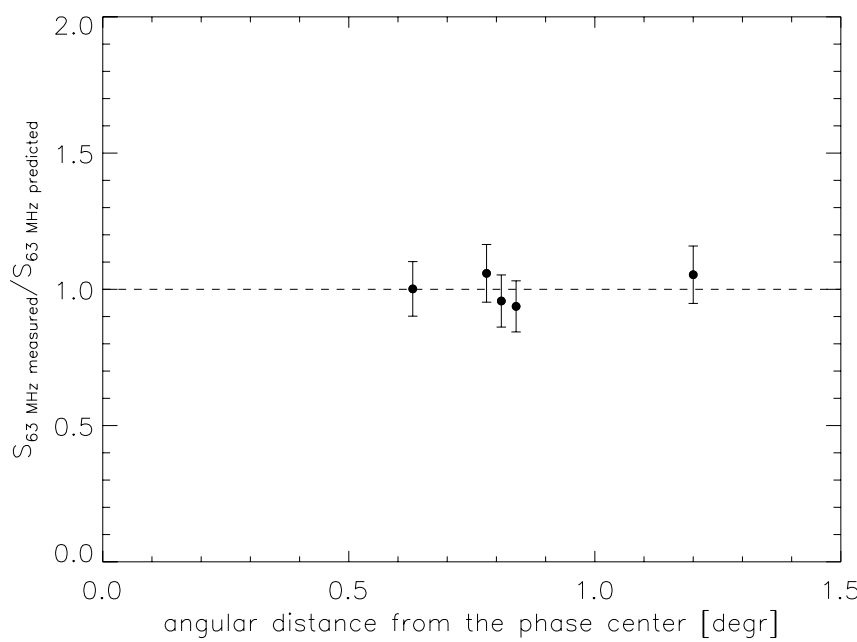

Fig. 3. Measured LOFAR fluxes and predicted fluxes for 5 bright sources in the A2256 field. For each of these sources, 8C, VLSS, 7C, WENSS, and NVSS flux densities were available. We used second order polynomial fits in $\log (S)-\log (v)$ space to compute the predicted $63 \mathrm{MHz}$ fluxes. An overall scaling factor of 1.23 was applied to the LOFAR fluxes. The error bars display the adopted $10 \%$ uncertainty in the flux scale.

\section{Results}

\subsection{Radio images}

An overview image of the field around A2256 is shown in Fig. 6. The $63 \mathrm{MHz}$ images are displayed in Fig. 7 and the 20 and $30 \mathrm{MHz}$ images in Fig. 8. We labelled some of the known sources in the cluster following the scheme from Bridle \& Fomalont (1976); Röttgering et al. (1994); van Weeren et al. (2009). The LOFAR $63 \mathrm{MHz}$ image reveals some of the wellknown tailed radio sources $(A, B, F)$, the main relic $(\mathrm{G}$ and $H)$, and part of the radio halo. A hint of the long and straight headtail source $\mathrm{C}$ is also visible.

The main radio relic and halo are somewhat better visible in the lower resolution $63 \mathrm{MHz}$ image (Fig. 7, right panel). Interestingly, the LOFAR image also reveals source $\mathrm{AG}+\mathrm{AH}$. This source has only been discovered recently (van Weeren et al. 2009) and is not visible in the deep VLA 1.4 GHz observations (Clarke \& Enßlin 2006), implying a steep spectrum. We do not detect the steep spectrum source AI, but this is expected since the integrated flux of this source is about a factor of two lower than $\mathrm{AG}+\mathrm{AH}$ at $325 \mathrm{MHz}$. The $30 \mathrm{MHz}$ image reveals source $\mathrm{F}$ and the combined emission from $\mathrm{A}$ and $\mathrm{B}$. The relic is also detected. At $20 \mathrm{MHz}$ the ionospheric phase distortions are quite severe, causing the relic to partly blend with source $F$ and the $\mathrm{A}+\mathrm{B}$ complex.

An overlay of the $63 \mathrm{MHz}$ image with a VLA $1.4 \mathrm{GHz}$ image is shown in Fig. 9. For this we combined the 1369, 1413, 1513, and $1703 \mathrm{MHz}$ VLA C-array images from Clarke $\&$ Enßlin (2006). These images were convolved to a common resolution of $16^{\prime \prime} \times 16^{\prime \prime}$ and then combined, adopting a flux scaling according to a spectral index of $\alpha=-1$. It is interesting to note the differences between the VLA and LOFAR LBA image around the $\mathrm{A}+\mathrm{B}$ complex. The VLA image peaks in brightness at the heads of the head-tail sources, while the LOFAR image mainly shows the tails. The steep spectrum B2 region, noticed by Intema (2009), also clearly stands out. A Chandra X-ray overlay is shown in Fig. 10. The X-ray emission in elongated in the NW-SE direction, with the relic located on the NW edge of the 

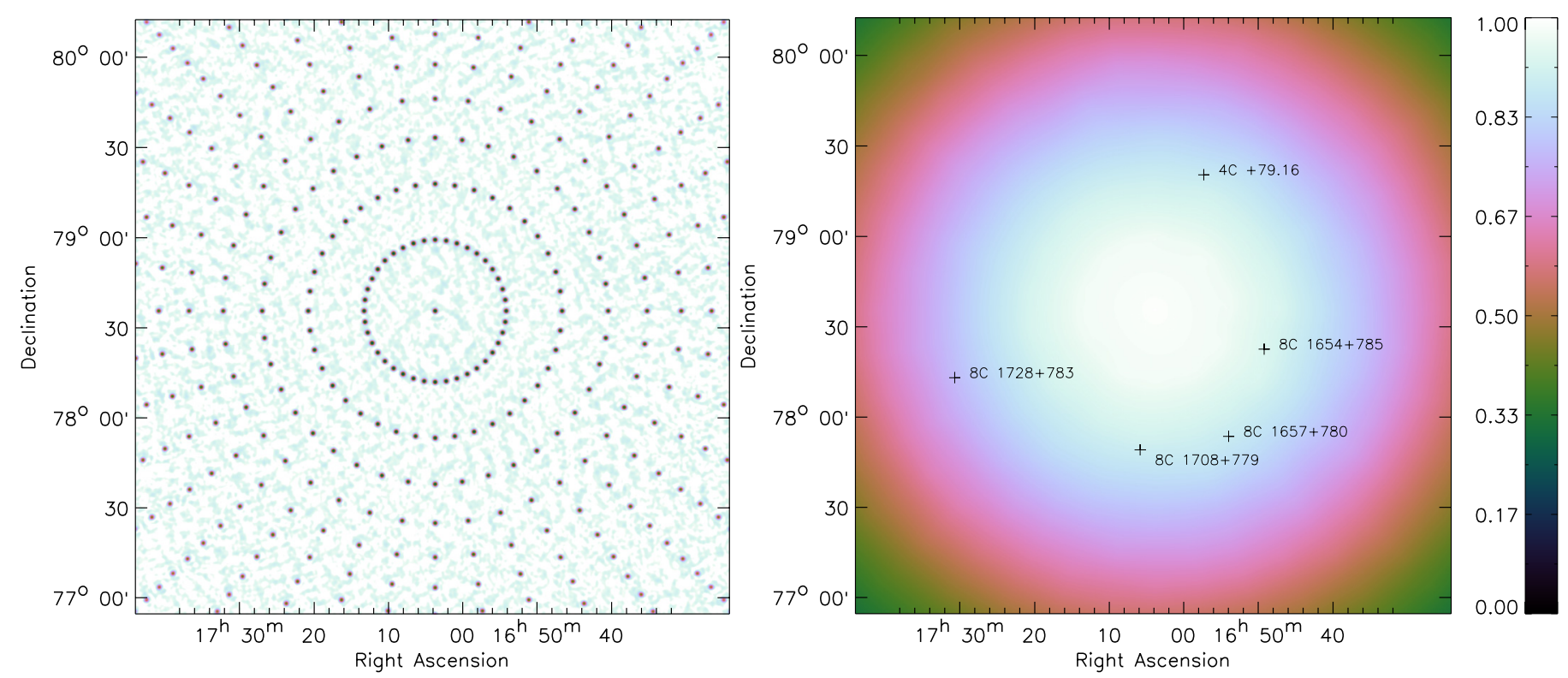

Fig. 4. Left: image of the simulated $1 \mathrm{Jy}$ point sources in the A2256 field at $63 \mathrm{MHz}$. Note that the background is not precisely zero because of deconvolution errors. The apparent flux of the sources decreases radially away from the phase center due to the primary beam attenuation. Color-scale is the same as in the right panel but inverted. Right: primary beam attenuation of the A2256 observations at $63 \mathrm{MHz}$ obtained by fitting a surface to the integrated fluxes of the sources in the left panel. The location of sources that were used to obtain the flux-scale are marked with crosses.

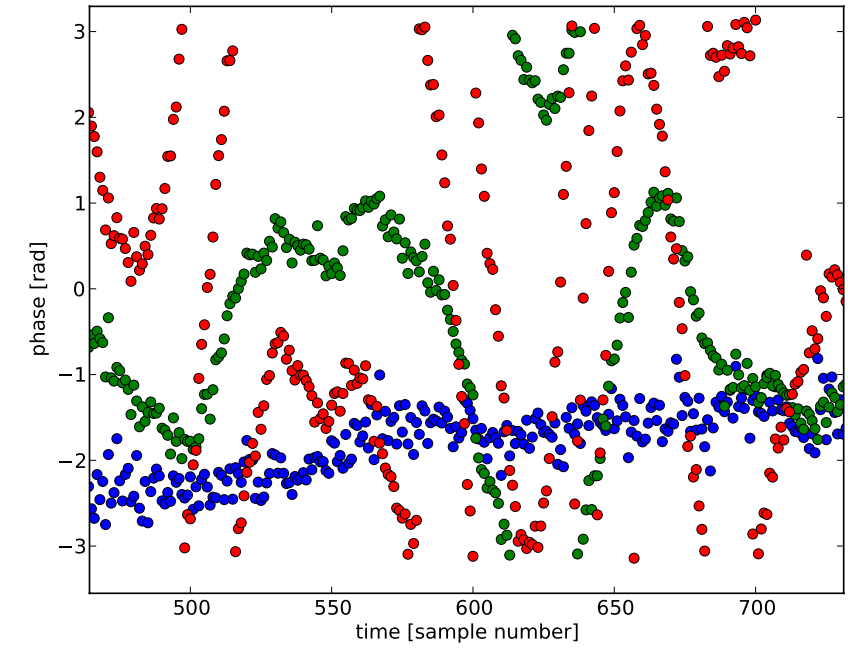

Fig. 5. Phase self-calibration solutions for stations CS017 (blue), RS205 (green), RS208 (red) at $63.9 \mathrm{MHz}$. Each time sample corresponds to $10 \mathrm{~s}$ and the phases are plotted relative to station CS003. The distances between the stations CS017, RS205, RS208 and core station CS003 are about $0.5,7$, and $29 \mathrm{~km}$, respectively.

X-ray emission. The two main X-ray peaks are located between source D and A and just north of source A.

\subsection{Spectral index map and integrated fluxes}

We made a spectral index map between 63 and $351 \mathrm{MHz}$, making use of the WSRT $351 \mathrm{MHz}$ map from Brentjens (2008). The WSRT map was made with uniform weighting of the visibilities and a Gaussian taper was applied in the uv-plane to create a circular 67" beam. We also made a LOFAR image with uniform weighting and a Gaussian taper to match the WSRT resolution of $67^{\prime \prime} \times 67^{\prime \prime}$. We applied an inner uv-range cut to the LOFAR data to match up the inner uv-range limit of the WSRT data. To increase the SNR per beam for the diffuse emission we convolved both images to $100^{\prime \prime}$ resolution, pixels below $3 \sigma_{\text {rms }}$ were blanked. The LOFAR-WSRT spectral index map and the corresponding spectral index error map are shown in Fig. 11.

The spectral index map reveals that the relic has a relatively flat spectral index of about -0.8 , with variations of 0.3 in $\alpha$ across the structure. For those parts of the radio halo where the spectral index can be measured, we find $\alpha$ to be in the range -1.0 to -1.7 . For source $F$ the spectral index is around -1.0 . For the combined emission from $\mathrm{AH}$ and $\mathrm{AG}$ we find $\alpha$ to be between -2.2 and -1.7 .

We extracted the integrated fluxes for sources in the cluster from the $63 \mathrm{MHz}$ image, see Table 2. The integrated fluxes of the radio halo and relic (source $\mathrm{G}+\mathrm{H}$ ) are difficult to measure because they are partly blended with some of the complex head-tail radio sources in the cluster. To estimate their flux contribution, we used both the high and low resolution images (Fig. 7). From the high resolution image we measured the fluxes for source $\mathrm{F}$, and the combined emission from $\mathrm{A}$ and $\mathrm{B}(\mathrm{A}+\mathrm{B})$. Head-tail source $\mathrm{C}$ contributes a significant amount of flux in the region of the relic, as judged from the GMRT $153 \mathrm{MHz}$ image from Intema (2009). In the $63 \mathrm{MHz}$ image the source blends with the radio relic, making it impossible to obtain a reliable flux estimate. Using the $153 \mathrm{MHz}$ GMRT image (giving a flux of $0.48 \pm 0.05 \mathrm{Jy}$ ) and the reported $327 \mathrm{MHz}$ VLA flux $(0.247 \pm 0.020 \mathrm{Jy}$; Röttgering et al. 1994) we find $\alpha=$ $-0.87 \pm 0.17$ for source C. Extrapolating this to $63 \mathrm{MHz}$, we estimate a flux of $1.05 \mathrm{Jy}$. To measure the relic flux we summed the flux over the same region as indicated in Fig. 10 by Brentjens (2008). After subtracting the flux contribution for source $C$, we obtain a relic flux of $5.6 \pm 0.8 \mathrm{Jy}$. A power-law fit through the relic's flux measurements at 1369 (Clarke \& Enßlin 2006), 351 (Brentjens 2008), 153 (Intema 2009), and $63 \mathrm{MHz}$ gives a spectral index of $-0.81 \pm 0.03$, see Fig. 13. For the radio halo we find a flux of $6.6 \pm 1.3 \mathrm{Jy}$ from Fig. 7 (right panel), integrating over the entire halo area as defined by the $351 \mathrm{MHz}$ image. 


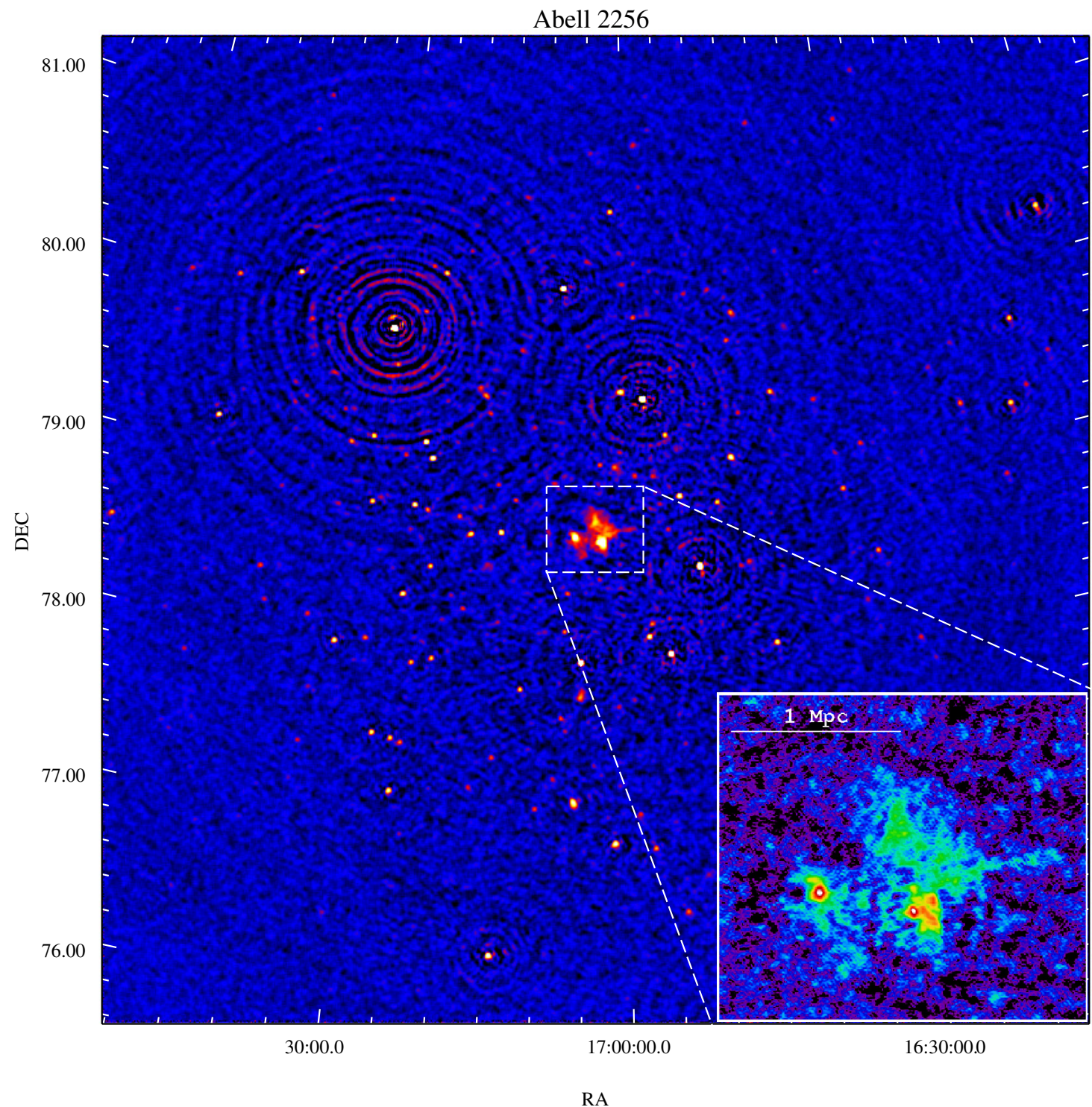

Fig. 6. Overview of the A2256 field at 61-67 MHz as observed with LOFAR. The image was not corrected for the primary beam attenuation and has a resolution of about $80^{\prime \prime}$. Bottom right: zoomed version of the 61-67 MHz image with a synthesized beam of $22^{\prime \prime} \times 26^{\prime \prime}$.

Table 2. $63 \mathrm{MHz}$ source fluxes and spectral indices computed between 63 and $153 \mathrm{MHz}$.

\begin{tabular}{|c|c|c|}
\hline Source & $\underset{\text { Jy }}{S_{63 \mathrm{MHz}}}$ & $\alpha_{63}^{153}$ \\
\hline $\mathrm{A}+\mathrm{B}$ & $5.1 \pm 0.6$ & $-1.1 \pm 0.2$ \\
\hline $\mathrm{F}$ & $2.8 \pm 0.3$ & $-1.2 \pm 0.2$ \\
\hline $\mathrm{AG}+\mathrm{AH}$ & $0.75 \pm 0.10$ & $-2.3 \pm 0.4$ \\
\hline relic $(\mathrm{G}+\mathrm{H})$ & $5.6 \pm 0.8$ & $-0.81 \pm 0.03^{a}$ \\
\hline halo & $6.6 \pm 1.3$ & $-1.5 \pm 0.1^{b}$ \\
\hline
\end{tabular}

Notes. ${ }^{(a)}$ From a polynomial fit in $\log (S)-\log (v)$ space, see Fig. 13. (b) Between 63 and $351 \mathrm{MHz}, \alpha$ taken from Fig. 13.

\section{Discussion}

The results on the radio spectra for source $\mathrm{F}, \mathrm{AG}+\mathrm{AH}$, the radio relic, and the radio halo are discussed in the following subsections.

\subsection{Source $F$}

Source F is known for its complex Z-shape morphology, see Fig. 9, and steep radio spectrum (e.g., Bridle et al. 1979). The source comprises three smaller structures: F1, F2, and F3. The brightest component F2, has a toroidal filamentary shape (Röttgering et al. 1994; Miller et al. 2003; Intema 2009). F3 has been classified as a head-tail radio source associated with galaxy 122 (named by Fabricant et al. 1989). No optical counterparts have been found for F1 and F2, and their origin is still being debated. Bridle et al. (1979) suggested that all three components are the tail of galaxy 122, this scenario is also discussed by Brentjens (2008). In another scenario, F2 could be the compressed fossil radio plasma from previous episodes of AGN activity (Enßlin \& Gopal-Krishna 2001; Enßlin \& Brüggen 2002). This agrees with the observed toroidal shape (Enßlin \& Brüggen 2002). In this case, the fossil plasma could also have originated from galaxy 122 .

We collected flux density measurements for F2 from the literature (see Brentjens 2008), these are plotted in Fig. 12. The 

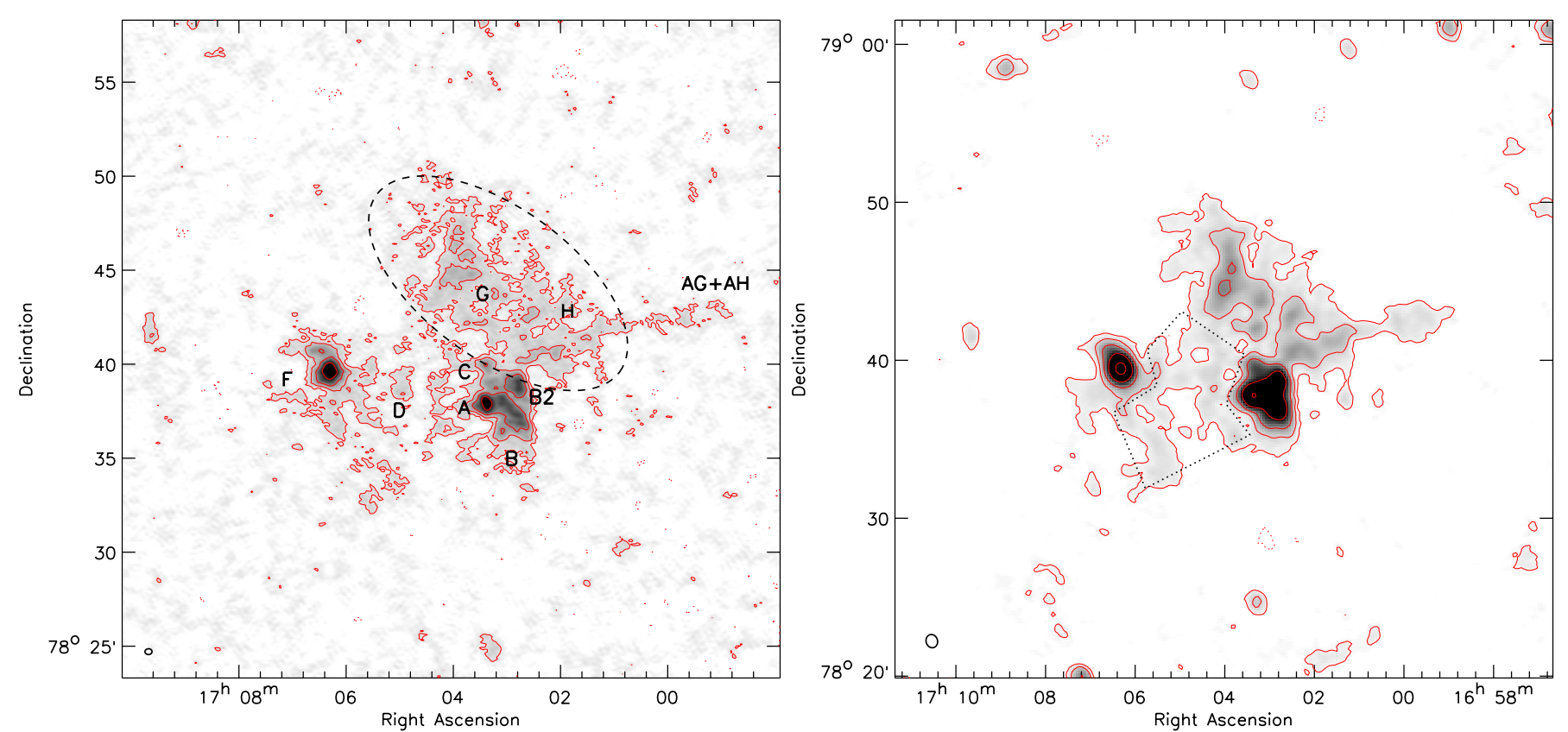

Fig. 7. A2256 61-67 MHz images represented in grey scale and contour form. The synthesized beams are shown in the bottom left corner. Left: high-resolution, $22^{\prime \prime} \times 26^{\prime \prime}$, image made with robust $=-0.1$ weighting. The radio relic (source $\mathrm{G}$ and $\mathrm{H}$ ) is indicated with the dashed ellipse. Contour levels are drawn at $[1,2,4, \ldots] \times 30 \mathrm{mJy}^{\text {beam }}{ }^{-1}$. Negative $3 \sigma_{\mathrm{rms}}$ contours are shown by the dotted lines. Right: low-resolution image, $52^{\prime \prime} \times 62^{\prime \prime}$, image made with robust $=0.5$ weighting. The area for the halo spectral index measurement is indicated by the dotted lines. Contour levels are drawn at $[1,2,4, \ldots] \times 75 \mathrm{mJy}^{\text {beam }}{ }^{-1}$.
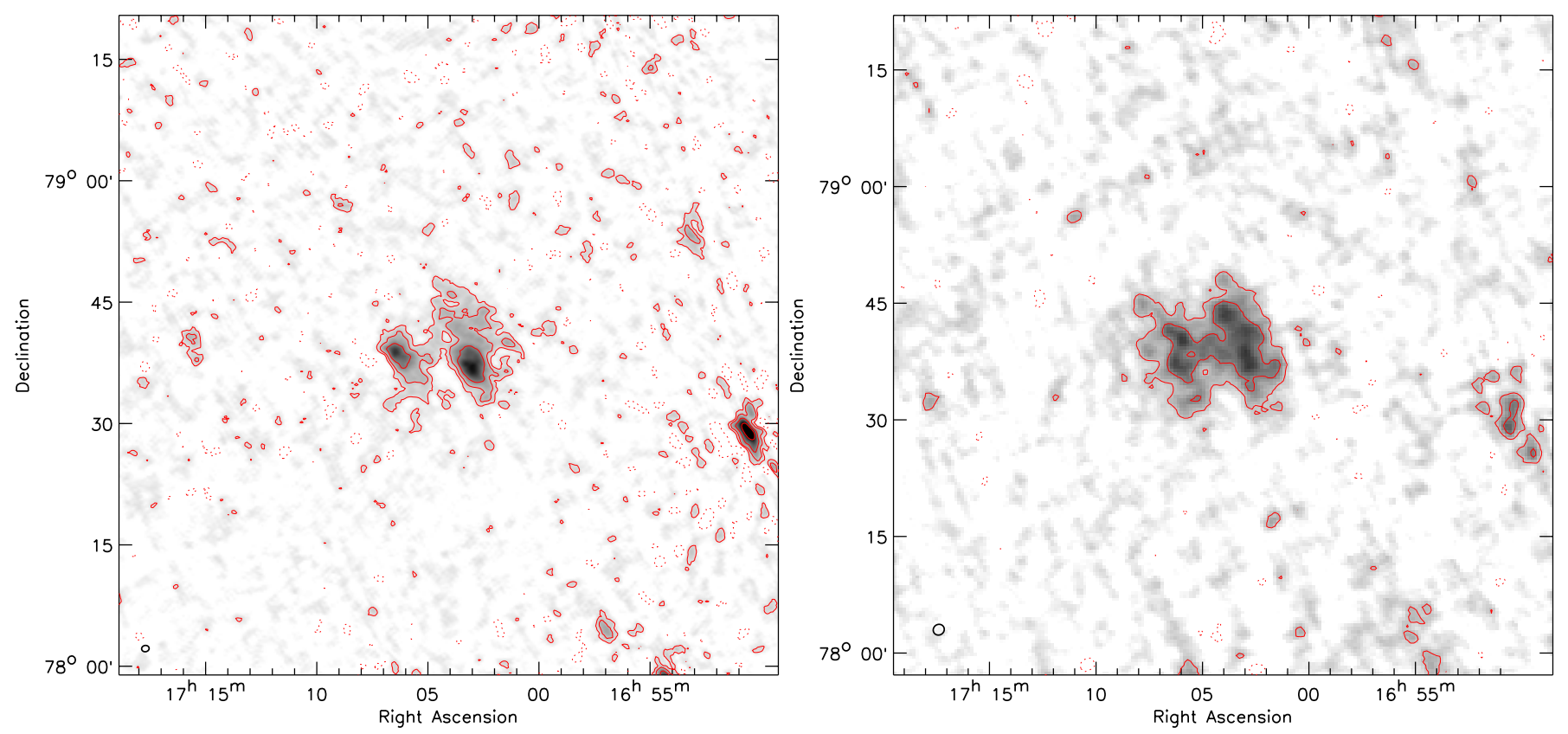

Fig. 8. A2256 30 and $20 \mathrm{MHz}$ images represented in grey scale and contour form. The synthesized beams are shown in the bottom left corner. Left: 28-32 MHz image with a resolution of $58^{\prime \prime} \times 69^{\prime \prime}$. Contour levels are drawn at $[1,2,4, \ldots] \times 129 \mathrm{mJy}$ beam ${ }^{-1}$. Negative $3 \sigma_{\text {rms }}$ contours are shown by the dotted lines. Right: $18-22 \mathrm{MHz}$ image with a resolution of $108^{\prime \prime} \times 116^{\prime \prime}$. Contour levels are drawn at $[1,2,4, \ldots] \times 0.75 \mathrm{Jy}^{\mathrm{beam}}{ }^{-1}$. Both images were made with robust $=0.1$ weighting. The bright compact source to the west around RA $16^{\mathrm{h}} 51^{\mathrm{m}}$ is $8 \mathrm{C} 1654+785$.

spectrum for F2 is clearly curved, with the spectrum flattening towards lower frequencies. From the fit, see Fig. 12, we find a spectral index of -0.95 between 63 and $153 \mathrm{MHz}$. Between 150 and $350 \mathrm{MHz}$ Kale \& Dwarakanath (2010) reported $\alpha=$ $-1.10 \pm 0.05$, but no flux density measurements were reported for F2. We find a spectral index of -1.34 between 150 and $350 \mathrm{MHz}$ using the polynomial fit. The reason for the difference is unclear, but it should be noted that the measurements from the literature are not consistent within their reported uncertainties. Between 610 and $1400 \mathrm{MHz}$ we obtain $\alpha=-1.67$, much steeper than at low-frequencies. In one scenario, Brentjens (2008) estimated a possible break frequency to be located at $26 \mathrm{MHz}$, assuming a constant magnetic field of $7.3 \mu \mathrm{G}$ and a spectral age of 0.2 Gyr. The $63 \mathrm{MHz}$ flux density measurement indicates 
R. J. van Weeren et al.: LOFAR observations of cluster-scale non-thermal radio emission in Abell 2256

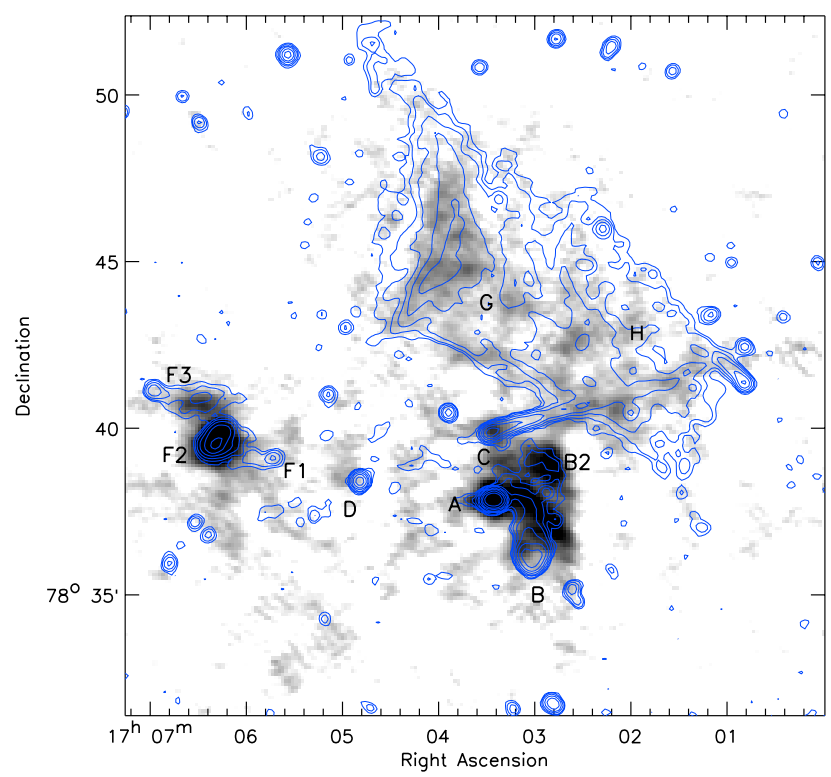

Fig. 9. LOFAR LBA $63 \mathrm{MHz}$ image overlaid with VLA $1.4 \mathrm{GHz}$ $\mathrm{C}$-array contours. The contour levels are drawn at $[1,2,4,8, \ldots] \times$

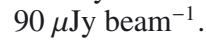

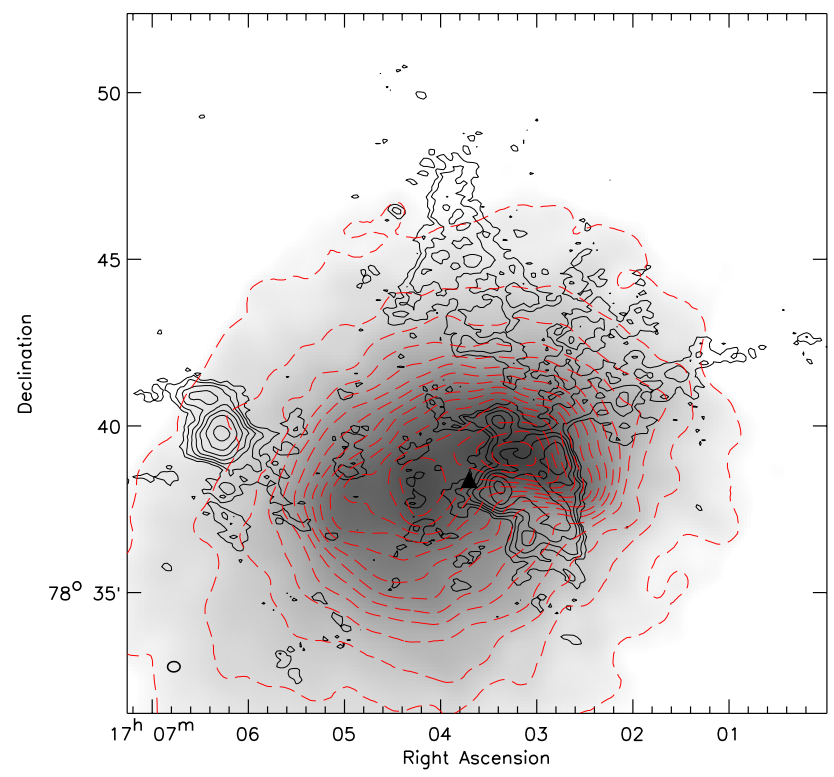

Fig. 10. Chandra $0.5-7 \mathrm{keV}$ ACIS-I/S co-added image smoothed by a variable-width Gaussian, Fig. 1 from Sun et al. (2002). Red dashed linearly spaced contours trace the X-ray isophotes. The high-resolution $63 \mathrm{MHz}$ LOFAR image is overlaid with black contours drawn at levels of $[1,2,4,8, \ldots] \times 35$ mJy beam $^{-1}$. The synthesized beam is shown in the bottom left corner. The triangle indicates the position of the main peak in the projected galaxy distribution derived by Ramella et al. (2007).

the spectrum continues to flatten. However, high-resolution measurements below $60 \mathrm{MHz}$ are needed to determine the possible underlying ("zero ageing") power-law component. The origin of source F2 remains unclear, although it is likely the source is somehow related to the fossil radio plasma from previous phases of AGN activity given its brightness and steep radio spectrum.

\subsection{Source $A G+A H$}

The combined emission from source $\mathrm{AG}+\mathrm{AH}$ is detected in the LOFAR $63 \mathrm{MHz}$ image. With the non-detection of these
Table 3. Spectral age of the source $\mathrm{AG}+\mathrm{AH}$ in Gyr for different break frequencies and magnetic field strengths.

\begin{tabular}{lcccc}
\hline \hline & $B=3 \mu \mathrm{G}$ & $B=5 \mu \mathrm{G}$ & $B=10 \mu \mathrm{G}$ & $B=20 \mu \mathrm{G}$ \\
\hline$v_{\text {brk }}=50 \mathrm{MHz}$ & 0.28 & 0.21 & 0.10 & 0.039 \\
$v_{\text {brk }}=20 \mathrm{MHz}$ & $\mathbf{0 . 4 4}$ & 0.33 & 0.16 & 0.061 \\
$v_{\text {brk }}=5 \mathrm{MHz}$ & $\mathbf{0 . 8 8}$ & $\mathbf{0 . 6 6}$ & 0.32 & 0.12 \\
$v_{\text {brk }}=1 \mathrm{MHz}$ & $\mathbf{2 . 0}$ & $\mathbf{1 . 5}$ & $\mathbf{0 . 7 1}$ & 0.27 \\
\hline
\end{tabular}

Notes. Values in boldface are sufficient to explain the distance of at least $800 \mathrm{kpc}$ between the head of the source $\mathrm{C}$ and $\mathrm{AG}+\mathrm{AH}$, in the scenario that $\mathrm{AG}+\mathrm{AH}$ is a part of the tail of $\mathrm{C}$.

sources in the deep $1.4 \mathrm{GHz}$ image from Clarke \& Enßlin (2006), van Weeren et al. (2009) determined that $\alpha<-1.95$ between 325 and $1369 \mathrm{MHz}$. Between 140 and $351 \mathrm{MHz}$, using WSRT observations, the spectral index is $-2.05 \pm 0.14$ at a resolution of $175^{\prime \prime}$. At this resolution the flux of this feature partly blends with the relic emission. With the $153 \mathrm{MHz}$ image from Intema (2009) we find a flux of $95 \pm 10 \mathrm{mJy}$ for the combined emission from $\mathrm{AG}+\mathrm{AH}$. From the GMRT $325 \mathrm{MHz}$ image a flux of $19 \pm 2$ mJy was reported (van Weeren et al. 2009). This gives a spectral index of $-2.1 \pm 0.2$ between these two frequencies, in agreement with the earlier reported result of $-2.05 \pm 0.14$. The LOFAR flux is $0.75 \pm 0.10 \mathrm{Jy}$ for this source. This results in a spectral index of $-2.3 \pm 0.4$ between 63 and $153 \mathrm{MHz}$. At low-frequencies the spectrum thus remains steep, although the uncertainty in the spectral index is too large to rule out a spectral turnover.

We consider the possibility that this emission is related to the head-tail source C. Following Miley (1980) and Brentjens (2008) for a constant magnetic field and no adiabatic losses, the radiative lifetime/spectral age $(\tau)$ is given as

$\tau=\frac{2.6 \times 10^{10}}{B^{2}+B_{\mathrm{CMB}}^{2}} \sqrt{\frac{B}{(1+z) v_{\mathrm{brk}}}}[\mathrm{yr}]$,

where $B$ is the magnetic field strength in $\mu \mathrm{G}, B_{\mathrm{CMB}}[\mu \mathrm{G}]=$ $3.25(1+z)^{2}$ the equivalent magnetic field strength of the microwave background, and $v_{\text {brk }}$ the break frequency in MHz. The LOFAR $63 \mathrm{MHz}$ flux density measurement indicates that $v_{\text {brk }}$ is located $\lesssim 50 \mathrm{MHz}$ because the spectrum is still very steep between 63 and $153 \mathrm{MHz}$. The magnetic field strength is difficult to estimate as the spectral shape is poorly constrained. With a reasonable value of $10 \mu \mathrm{G}$ we obtain a spectral age of $0.1 \mathrm{Gyr}$. This increases to $0.2 \mathrm{Gyr}$ for $B=5 \mu \mathrm{G}$. In all cases we assumed $\nu_{\text {brk }}=50 \mathrm{MHz}$. Spectral ages for different break frequencies and magnetic field values are listed in Table 3. Röttgering et al. (1994) estimated a velocity $(v)$ of $\sim 2000 \mathrm{~km} \mathrm{~s}^{-1}$ for the head-tail source. Then the separation between the "head" and AG+AH would be $v \times \tau=200 \mathrm{kpc}$, for $B=10 \mu \mathrm{G}$. AG+AH is located at a projected distance of about $800 \mathrm{kpc}$ from the head of C. A lower magnetic field strength of $B=3.0 \mu \mathrm{G}$ would give a distance of $600 \mathrm{kpc}$. This is still lower than the projected distance of $800 \mathrm{kpc}$ between the head of $\mathrm{C}$ and $\mathrm{AG}+\mathrm{AH}$. Therefore $v_{\mathrm{brk}}$ could be located at a lower frequency than $50 \mathrm{MHz}$ (Table 3). If the radio plasma from the tail (the $\mathrm{AG}+\mathrm{AH}$ part) is compressed by the merger shock wave, the radiative age of the source can also be older (about 0.5 Gyr or more, e.g., Dwarakanath \& Kale 2009; Kale \& Dwarakanath 2012). In this case Eq. (1) does not apply. This makes it easier to explain the distance of at least $800 \mathrm{kpc}$, even more since this distance is a lower limit because of unknown projection effects. Given all these uncertainties, source $\mathrm{AG}+\mathrm{AH}$ could indeed be an old part of the tail of source $\mathrm{C}$. 

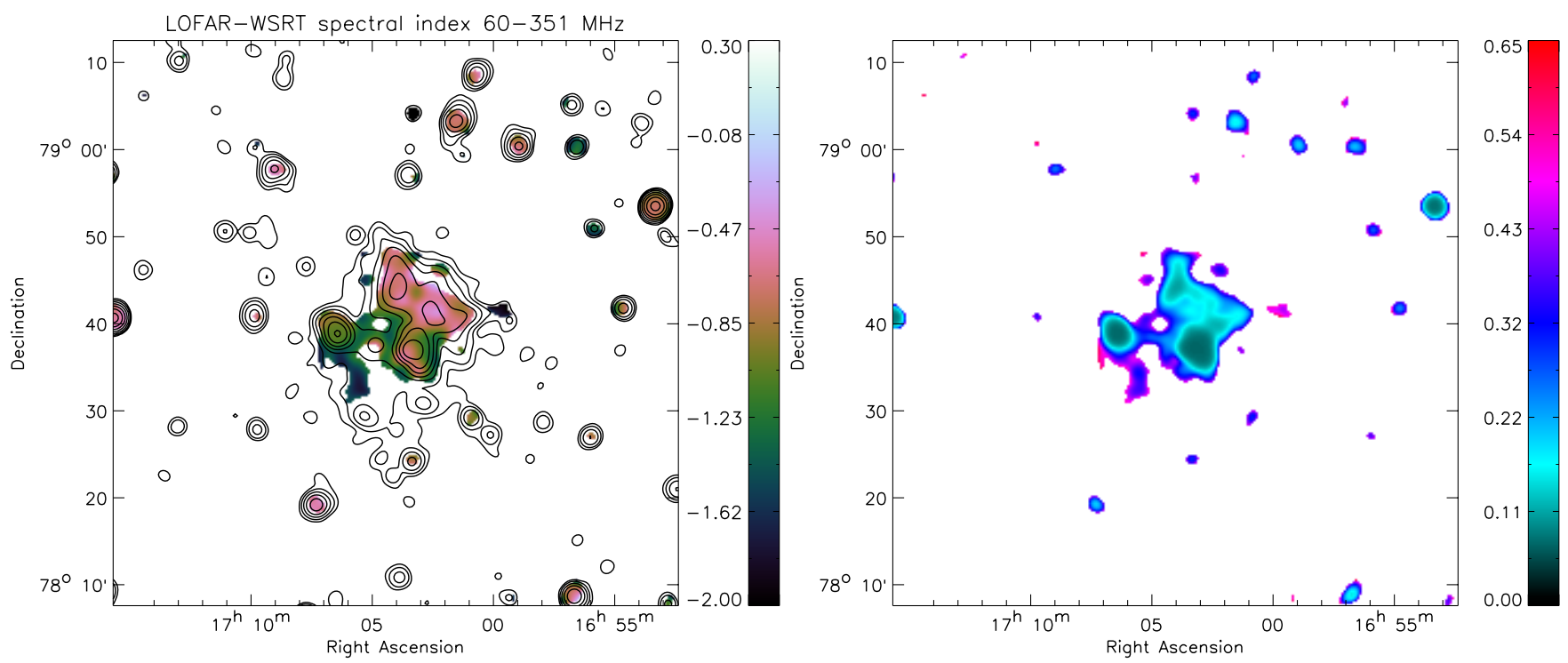

Fig. 11. Left: LOFAR LBA - WSRT spectral index map between 63 and $351 \mathrm{MHz}$. Total intensity contours at $351 \mathrm{MHz}$ are shown at levels of $[1,2,4,8, \ldots] \times 1.0 \mathrm{mJy}_{\text {beam }}^{-1}$. The $351 \mathrm{MHz}$ image is taken from Brentjens (2008). Right: spectral index error map. The map is computed on the basis of the $\sigma_{\text {rms }}$ values for the individual maps and the reported flux calibration uncertainty of $10 \%$.

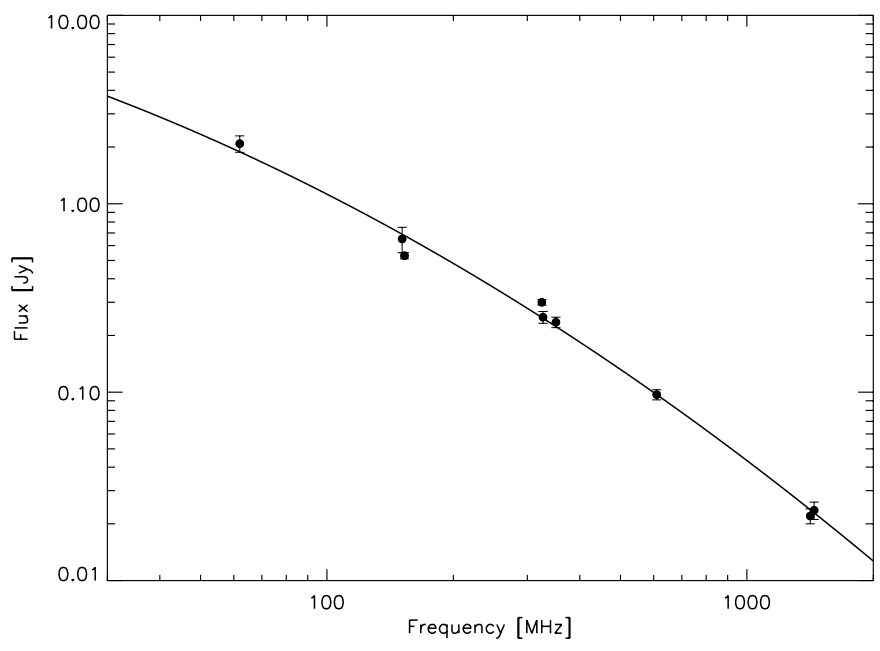

Fig. 12. Radio spectrum of F2. The fluxes were taken from Brentjens (2008), except for the $63 \mathrm{MHz}$ LOFAR flux. The solid line is a second order polynomial fit through the flux density measurements in $\log (S)-\log (v)$ space. During the fitting procedure, we assumed $10 \%$ uncertainty because the flux density measurements are not all consistent within their $1 \sigma$ reported errors (plotted in the figure). This prevents the fit to be forced to go through a few measurements with small reported uncertainties.

\subsection{Radio relic}

Radio gischt relics are proposed to trace particle acceleration (or re-acceleration of seed particles) at shocks. In the linear test particle regime and assuming that particles can diffuse across the shock discontinuity (DSA), the spectrum of the re-accelerated particles is given by (e.g., Blandford \& Eichler 1987):

$N(p)=\left(-\delta_{\text {inj }}+2\right) p^{\delta_{\mathrm{inj}}} \int_{p_{\min }}^{p_{\max }} N_{\mathrm{s}}(p) p^{-\delta_{\mathrm{inj}}-1} \mathrm{~d} p$

where $N_{\mathrm{s}}(p)$ is the spectrum of seed particles and

$\delta_{\text {inj }}=-2 \frac{\mathcal{M}^{2}+1}{\mathcal{M}^{2}-1}$
$\mathcal{M}$ is the Mach number of the shock. The corresponding synchrotron spectral index, not including energy losses, is $\alpha_{\text {inj }}=\left(\delta_{\text {inj }}-1\right) / 2$ (with $\left.F(v) \propto v^{\alpha_{\text {inj }}}\right)$.

If the properties of the shock remain unchanged (i.e., "stationary conditions"), the relic has been present in the ICM longer than the electron cooling time and the cooling time is shorter than the diffusion time, the integrated radio spectrum will be a power-law, with a spectral index that is about 0.5 units steeper than $\alpha_{\text {inj }}$ (Kardashev 1962). The radio spectrum of the A2256 relic has a power-law shape over the observed frequency range, with a spectral index of $-0.81 \pm 0.03$. This would imply $\alpha_{\text {inj }} \sim-0.3$ for a simple shock model. The flattest possible injection spectral index is -0.5 from DSA, which is not consistent with $\alpha_{\text {inj }} \sim-0.3$. Therefore this directly implies that stationary conditions for the shock do not apply and/or the acceleration process is more complicated ${ }^{5}$. It could suggest that the relic brightened very recently $(<0.1 \mathrm{Gyr}$ or so) due to an increase in the Mach number while the shock front propagated outward, since the efficiency of DSA is believed to increase strongly with Mach number (e.g., Hoeft \& Brüggen 2007, at least in this regime with $\mathcal{M} \lesssim 4$ ). Recently injected relativistic electrons would thus have a higher density and hence a higher brightness. Therefore, the average spectral slope below the flattest value expected from standard DSA may reflect the strengthening of the shock front while moving outwards. In this case no equilibrium has yet been reached between the electron cooling and injection within the observed frequency range below $1369 \mathrm{MHz}$, but one expects spectral steepening at higher frequencies. Clarke \& Enßlin (2006) indeed reported a steeper spectral index of -1.2 between 1369 and $1703 \mathrm{MHz}$ for the relic. For a typical magnetic field strength of $2 \mu \mathrm{G}$ for the relic (Clarke \& Enßlin 2006) and $v_{\text {brk }}=1400 \mathrm{MHz}$, the spectral age is about 0.05 Gyr.

Berrington et al. (2002) proposed that A2256 is undergoing a triple merger event. One between a subcluster and the primary cluster and one between the primary cluster and subcluster

\footnotetext{
5 The electron cooling time can also not be longer than the diffusion time. At $1.4 \mathrm{GHz}$, where the relic has about the same size as at $63 \mathrm{MHz}$, the electron cooling time is orders magnitude smaller than the diffusion time (e.g., Jaffe 1977; Bagchi et al. 2002).
} 
(or the primary cluster only) with a smaller galaxy group. The relative velocity between the subcluster and primary cluster is estimated to be $\sim 2000 \mathrm{~km} \mathrm{~s}^{-1}$ and they are near the time of the first close passage of the subcluster and primary cluster centers. The group plunges down from the north. The merger between the primary cluster and subcluster has a mass ratio of about 3 and the merger between the primary cluster (+subcluster) and group has a mass ratio of about 10. Miller et al. (2003) argued that the "primary cluster-galaxy group" merger is responsible for the radio relic and that the merger event is viewed 0.3 Gyr after the core passage. From the radio spectra alone it is not possible to disentangle the merger scenario, but usually the strongest shocks form after core passage (Vazza et al. 2012) and the flat integrated radio spectrum implies that the relic only recently brightened. Further flux density measurements above $2 \mathrm{GHz}$ are needed to better constrain the high-frequency end of the spectrum and confirm the radio spectrum steepens here.

No shock has been found so far in X-ray observations (e.g., Bourdin \& Mazzotta 2008). Although, given the large extent of the relic in both the NS and EW directions we are probably not viewing the relic close to edge-on, making it more difficult to detect a shock. For an edge-on shock/relic one would expect a much larger ratio between the largest physical extent and the relic width (e.g., van Weeren et al. 2010). The relic measures about 1 by $0.5 \mathrm{Mpc}$. We can estimate the viewing angle if we assume the relic traces a planar shock located in the $x y$-plane and has a similar extent in both the $x$ and $y$ directions and a negligible extent in the shock downstream region (compared to the observed width). With the ratio of the largest physical size to the relic width this gives a viewing angle of about $30^{\circ}$ from edgeon. This is consistent with the estimate from Enßlin et al. (1998), based on the polarization fraction, which indicates the relic is seen under an angle of less than $\sim 50^{\circ}$ from edge-on. The relic is located at a project distance of $\sim 400 \mathrm{kpc}$ from the optical center of the cluster (Fig. 10). If the relic is seen under a viewing angle of $30^{\circ}$ from edge-on it is located at a true distance of $\sim 0.5 \mathrm{Mpc}$ from the cluster center. This is about a factor of two closer to the cluster center than most of the double radio relic clusters (e.g., Röttgering et al. 1997; Bagchi et al. 2006; Bonafede et al. 2009; van Weeren et al. 2011b). Together with the flat radio spectrum this is consistent with the fact that the relic in A2256 is seen at a relatively early stage in the merger, approximately half of the time after core passage compared to some of the double radio relic clusters.

Due to the large size of the relic it is unlikely we are seeing fossil radio plasma compressed by a shock wave, since radiative energy losses during the time it takes to compress a several hundred kiloparsec sized radio ghost would remove most of the electrons responsible for the observable radio emission (Clarke \& Enßlin 2006). In addition, we would expect steep curved radio spectra due to synchrotron and IC losses.

The unusual flat spectrum of the relic may also suggest a more complex situation. A synchrotron spectral index $\alpha>$ $\alpha_{\text {inj }}-1 / 2$ is expected if electrons accelerated at the passage of the shock, with spectrum $N(p) \propto p^{-\delta_{\text {inj }}}$, are also re-accelerated by some other mechanism in the region downstream of the shock. Under particular conditions a flatter spectrum is also expected if the accelerated electrons cool/age in a inhomogeneous downstream region. Particle re-acceleration mechanisms downstream of large-scale shocks in galaxy clusters, possibly connected with the turbulence that could be generated by the shock passage, is suggested by the existence of bridges of faint radio emission that connect relics and halos in several systems (Markevitch 2010,

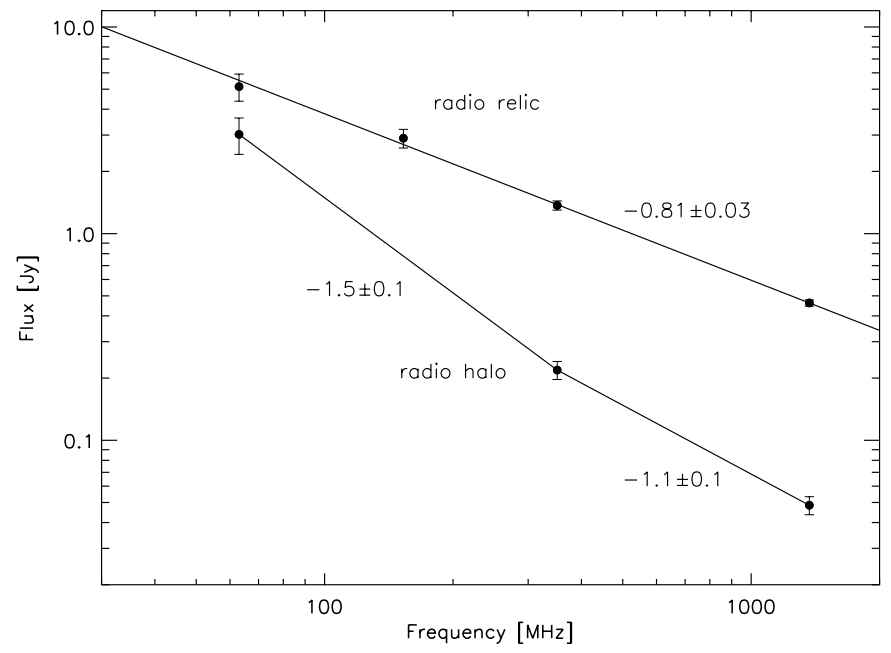

Fig. 13. Radio halo and relic spectrum. Flux density measurements from this work, Clarke \& Enßlin (2006), Brentjens (2008), and Intema (2009) were included. The solid straight line is a power-law fit to the relic fluxes. The radio halo spectrum is from flux density measurements summed over the region indicated in Fig. 7.

and references therein). In the case of A2256, projection effects may mix together the relic and bridge emission.

\subsection{Radio halo}

The spectral index of the radio halo was measured by summing the flux in the region around source D, using the imaged displayed in Fig. 7 (right panel). In this region, the halo is detected at the $1-3 \sigma$ level per beam in the LOFAR $63 \mathrm{MHz}$ image. We summed the flux over the same region in the $351 \mathrm{MHz}$ WSRT and $1369 \mathrm{MHz}$ VLA D-array (Clarke \& Enßlin 2006) images. The resulting radio spectrum is shown in Fig. 13. The $63-351 \mathrm{MHz}$ spectral index is $-1.5 \pm 0.1$ and the $351-1369 \mathrm{MHz}$ spectral index is $-1.1 \pm 0.1$. The low-frequency radio halo spectrum is steeper than at high-frequencies, although we only measured the spectrum in the area indicated in Fig. 7.

LOFAR observations at $63 \mathrm{MHz}$ clearly show a significant upturn of the spectrum of the radio halo at lower frequencies. This confirms and strengthens previous observational claims Kale \& Dwarakanath (2010) and provides totally new information for the interpretation of the origin of the halo. With only three data points it is premature to attempt a detailed modeling, this is matter for future papers when more data will be available. The complex spectrum may simply result from a superposition of two (or more) components, as previously suggested by Kale \& Dwarakanath (2010). Specifically, the emission of the relic could be projected on the halo emission causing a flattening of the spectrum at higher frequencies. We also note that recent modeling of turbulent re-acceleration of relativistic protons and their secondaries in the ICM predict a flattening of the synchrotron spectrum of radio halos at higher frequencies (Brunetti \& Lazarian 2011). In these models the flattening marks the transition between the spectral component due to turbulent re-acceleration and the underlying spectrum generated by the continuous injection of secondary electrons; the spectral shape of A2256 would constrain the turbulent acceleration time-scale $\simeq 0.5$ Gyr. Observations however may reveal situations more complex than those considered in the presently available models. Different populations of emitting electrons may coexist in the volume of the radio halo in the case they originate from multiple 
acceleration mechanisms, or the efficiency of their acceleration changes with space and time in the emitting volume. The latter hypothesis may reflect the fact that magnetic turbulence in a $\mathrm{Mpc}^{3}$ region of the ICM is not homogeneous. New observations with LOFAR at $150 \mathrm{MHz}$ and complementary observations at high frequencies are needed to determine the shape of the spectrum of the halo and to better constrain its origin.

\section{Conclusions}

We have presented initial results from LOFAR LBA observations between 18 and $67 \mathrm{MHz}$ of the cluster Abell 2256. We focussed mainly on the $63 \mathrm{MHz}$ map because at lower frequencies ionospheric phase distortions were severe. At $63 \mathrm{MHz}$ we detect both the radio halo and main radio relic. The radio spectrum for the relic is consistent with a power-law, with $\alpha=-0.81 \pm 0.03$. The integrated radio spectrum of the relic is quite flat, which could mean that the relic has only recently brightened due to an increase in the Mach number, within the last $\sim 0.1$ Gyr. Alternatively, particles are re-accelerated by some mechanism in the downstream region of the shock. For the radio halo we find low-frequency spectral steepening, that was previously also reported by Kale \& Dwarakanath (2010). Additional flux density measurements are needed to better determine the low-frequency spectrum of the halo and the mechanisms responsible for the acceleration of the emitting particles.

We detected a recently discovered steep spectrum source in the west of the cluster center, located roughly at the end of the previously known head-tail source C. For this source we find an extremely steep spectral index of $-2.3 \pm 0.4$ between 63 and $153 \mathrm{MHz}$. This steep spectrum source could be an older part of the tail of source C. For the source F2, we find that the spectral index flattens to -0.95 between 63 and $153 \mathrm{MHz}$. The origin of the source remains unclear.

In the future we plan to extend our investigation to lower frequencies. For this, ionospheric calibration schemes will be important to retain enough spatial resolution to separate the contribution from the various complex sources in the cluster.

Acknowledgements. We thank the anonymous referee for useful comments. LOFAR, the Low Frequency Array designed and constructed by ASTRON, has facilities in several countries, that are owned by various parties (each with their own funding sources), and that are collectively operated by the International LOFAR Telescope (ILT) foundation under a joint scientific policy. We thank Ming Sun for providing Chandra X-ray image. We have used the "cubehelix" color scheme from Green (2011). R.J.v.W. acknowledges funding from the Royal Netherlands Academy of Arts and Sciences. M.B., A.B. and M.H. acknowledge support by the Deutsche Forschungsgemeinschaft under grant FOR1254. G.B. and R.C. acknowledge partial support through PRIN-INAF 2009 and ASI-INAF I/009/10/0. C.F. and G.M. acknowledge financial support by the "Agence Nationale de la Recherche" through grant ANR09-JCJC-0001-01. Basic research in radio astronomy at the Naval Research Laboratory is supported by 6.1 Base funding. The Open University is incorporated by Royal Charter (RC 000391), an exempt charity in England $\&$ Wales and a charity registered in Scotland (SC 038302). HTI is Jansky Fellow of the National Radio Astronomy Observatory. O.W. is supported by the "Deutsche Forschungsgemeinschaft" (Emmy-Noether Grant WU 588/1-1) and by the European Commission (European Reintegration Grant PERG02-GA2007-224897 "WIDEMAP").

\section{References}

Bagchi, J., Enßlin, T. A., Miniati, F., et al. 2002, New Astron., 7, 249 Bagchi, J., Durret, F., Neto, G. B. L., \& Paul, S. 2006, Science, 314, 791 Berrington, R. C., Lugger, P. M., \& Cohn, H. N. 2002, AJ, 123, 2261
Bhatnagar, S., Cornwell, T. J., Golap, K., \& Uson, J. M. 2008, A\&A, 487, 419 Blandford, R., \& Eichler, D. 1987, Phys. Rep., 154, 1

Blandford, R. D., \& Ostriker, J. P. 1978, ApJ, 221, L29

Blasi, P., \& Colafrancesco, S. 1999, Astropart. Phys., 12, 169

Bonafede, A., Giovannini, G., Feretti, L., Govoni, F., \& Murgia, M. 2009, A\&A, 494, 429

Bourdin, H., \& Mazzotta, P. 2008, A\&A, 479, 307

Brentjens, M. A. 2008, A\&A, 489, 69

Bridle, A. H., \& Fomalont, E. B. 1976, A\&A, 52, 107

Bridle, A. H., Fomalont, E. B., Miley, G. K., \& Valentijn, E. A. 1979, A\&A, 80, 201

Briel, U. G., \& Henry, J. P. 1994, Nature, 372, 439

Briel, U. G., Henry, J. P., Schwarz, R. A., et al. 1991, A\&A, 246, L10

Briggs, D. S. 1995, Ph.D. Thesis, New Mexico Institute of Mining Technology, Socorro, New Mexico, USA

Brown, S., \& Rudnick, L. 2011, MNRAS, 412, 2

Brunetti, G. 2004, J. Korean Astron. Soc., 37, 493

Brunetti, G., \& Blasi, P. 2005, MNRAS, 363, 1173

Brunetti, G., \& Lazarian, A. 2011, MNRAS, 410, 127

Brunetti, G., Setti, G., Feretti, L., \& Giovannini, G. 2001, MNRAS, 320, 365

Brunetti, G., Giacintucci, S., Cassano, R., et al. 2008, Nature, 455, 944

Cassano, R., Brunetti, G., \& Setti, G. 2006, MNRAS, 369, 1577

Cassano, R., Brunetti, G., Röttgering, H. J. A., \& Brüggen, M. 2010a, A\&A, 509, A68

Cassano, R., Ettori, S., Giacintucci, S., et al. 2010b, ApJ, 721, L82

Clarke, T. E., \& Enßlin, T. A. 2006, AJ, 131, 2900

Cohen, A. S., Lane, W. M., Cotton, W. D., et al. 2007, AJ, 134, 1245

Condon, J. J., Cotton, W. D., Greisen, E. W., et al. 1998, AJ, 115, 1693

Cornwell, T. J., Golap, K., \& Bhatnagar, S. 2005, in Astronomical Data Analysis Software and Systems XIV, ed. P. Shopbell, M. Britton, \& R. Ebert, ASP Conf. Ser., 347, 86

Cornwell, T. J., Golap, K., \& Bhatnagar, S. 2008, IEEE J. Selected Topics in Signal Processing, 2, 647

Dallacasa, D., Brunetti, G., Giacintucci, S., et al. 2009, ApJ, 699, 1288

Dennison, B. 1980, ApJ, 239, L93

Dolag, K., \& Enßlin, T. A. 2000, A\&A, 362, 151

Dolag, K., Grasso, D., Springel, V., \& Tkachev, I. 2005, J. Cosmology Astropart. Phys., 1, 9

Dolag, K., Bykov, A. M., \& Diaferio, A. 2008, Space Sci. Rev., 134, 311

Donnert, J., Dolag, K., Brunetti, G., Cassano, R., \& Bonafede, A. 2010, MNRAS, 401, 47

Drury, L. O. 1983, Rep. Prog. Phys., 46, 973

Dwarakanath, K. S., \& Kale, R. 2009, ApJ, 698, L163

Ebeling, H., Edge, A. C., Bohringer, H., et al. 1998, MNRAS, 301, 881

Enßlin, T., Pfrommer, C., Miniati, F., \& Subramanian, K. 2011, A\&A, 527, A99

Enßlin, T. A., \& Brüggen, M. 2002, MNRAS, 331, 1011

Enßlin, T. A., \& Gopal-Krishna. 2001, A\&A, 366, 26

Enßlin, T. A., Biermann, P. L., Klein, U., \& Kohle, S. 1998, A\&A, 332, 395

Fabricant, D. G., Kent, S. M., \& Kurtz, M. J. 1989, ApJ, 336, 77

Feretti, L. 2005, Adv. Space Res., 36, 729

Ferrari, C., Govoni, F., Schindler, S., Bykov, A. M., \& Rephaeli, Y. 2008, Space Sci. Rev., 134, 93

Giacintucci, S., Venturi, T., Macario, G., et al. 2008, A\&A, 486, 347

Green, D. A. 2011, BASI, 39, 289

Hales, S. E. G., Waldram, E. M., Rees, N., \& Warner, P. J. 1995, MNRAS, 274, 447

Hales, S. E. G., Riley, J. M., Waldram, E. M., Warner, P. J., \& Baldwin, J. E. 2007, MNRAS, 382, 1639

Hamaker, J. P. 2011, Mathematical-physical analysis of the generic dual-dipole antenna, Tech. Rep., ASTRON

Heald, G., McKean, J., Pizzo, R., et al. 2010, in ISKAF2010 Science Meeting

Hoeft, M., \& Brüggen, M. 2007, MNRAS, 375, 77

Intema, H. T. 2009, Ph.D. Thesis, Leiden University, The Netherlands

Intema, H. T., van der Tol, S., Cotton, W. D., et al. 2009, A\&A, 501, 1185

Jaffe, W. J. 1977, ApJ, 212, 1

Jones, F. C., \& Ellison, D. C. 1991, Space Sci. Rev., 58, 259

Kale, R., \& Dwarakanath, K. S. 2010, ApJ, 718, 939

Kale, R., \& Dwarakanath, K. S. 2012, ApJ, 744, 46

Kang, H., \& Ryu, D. 2011, ApJ, 734, 18

Kardashev, N. S. 1962, SvA, 6, 317

Kassim, N. E., Lazio, T. J. W., Erickson, W. C., et al. 2007, ApJS, 172, 686

Kempner, J. C., Blanton, E. L., Clarke, T. E., et al. 2004, in The Riddle of Cooling Flows in Galaxies and Clusters of galaxies, ed. T. Reiprich, J. Kempner, \& N. Soker, 335

Lane, W. M., Cohen, A. S., Kassim, N. E., \& Lazio, T. J. W. 2005, in From Clark Lake to the Long Wavelength Array: Bill Erickson's Radio Science, ed. N. Kassim, M. Perez, W. Junor, \& P. Henning, ASP Conf. Ser., 345, 203

Macario, G., Venturi, T., Brunetti, G., et al. 2010, A\&A, 517, A43 
Macario, G., Markevitch, M. Giacintucci, S., et al. 2011, ApJ, 728, 82 Malkov, M. A., \& O'C Drury, L. 2001, Rep. Prog. Phys., 64, 429 Markevitch, M. 2010 [arXiv: 1010.3660]

Markevitch, M., Gonzalez, A. H., David, L., et al. 2002, ApJ, 567, L27

Markevitch, M., Govoni, F., Brunetti, G., \& Jerius, D. 2005, ApJ, 627, 733

Masson, C. R., \& Mayer, C. J. 1978, MNRAS, 185, 607

Miley, G. 1980, ARA\&A, 18, 165

Miller, N. A., Owen, F. N., \& Hill, J. M. 2003, AJ, 125, 2393

Miniati, F., Jones, T. W., Kang, H., \& Ryu, D. 2001, ApJ, 562, 233

Offringa, A. R., de Bruyn, A. G., Biehl, M., et al. 2010, MNRAS, 405, 155

Pandey, V. N., van Zwieten, J. E., de Bruyn, A. G., \& Nijboer, R. 2009, in The

Low-Frequency Radio Universe, ed. D. J. Saikia, D. A. Green, Y. Gupta, \&

T. Venturi, ASP Conf. Ser., 407, 384

Petrosian, V. 2001, ApJ, 557, 560

Pfrommer, C., \& Enßlin, T. A. 2004, MNRAS, 352, 76

Ramella, M., Biviano, A., Pisani, A., et al. 2007, A\&A, 470, 39

Rengelink, R. B., Tang, Y., de Bruyn, A. G., et al. 1997, A\&AS, 124, 259

Röttgering, H., Snellen, I., Miley, G., et al. 1994, ApJ, 436, 654

Röttgering, H. J. A., Wieringa, M. H., Hunstead, R. W., \& Ekers, R. D. 1997, MNRAS, 290, 577

Russell, H. R., Sanders, J. S., Fabian, A. C., et al. 2010, MNRAS, 406, 1721

Ruzmaikin, A., Sokolov, D., \& Shukurov, A. 1989, MNRAS, 241, 1

Struble, M. F., \& Rood, H. J. 1999, ApJS, 125, 35

Sun, M., Murray, S. S., Markevitch, M., \& Vikhlinin, A. 2002, ApJ, 565, 867

van Weeren, R. J., Intema, H. T., Oonk, J. B. R., Röttgering, H. J. A., \& Clarke, T. E. 2009, A\&A, 508, 1269

van Weeren, R. J., Röttgering, H. J. A., Brüggen, M., \& Hoeft, M. 2010, Science, 330,347

van Weeren, R. J., Brüggen, M., Röttgering, H. J. A., et al. 2011a, A\&A, 533, A35

van Weeren, R. J., Hoeft, M., Röttgering, H. J. A., et al. 2011b, A\&A, 528, A38

Vazza, F., Tormen, G., Cassano, R., Brunetti, G., \& Dolag, K. 2006, MNRAS, 369, L14

Vazza, F., Brüggen, M., van Weeren, R., et al. 2012, MNRAS, 421, 1868

Venturi, T. 2011, Mem. Soc. Astron. Itali., 82, 499

1 Leiden Observatory, Leiden University, PO Box 9513, 2300 RA Leiden, The Netherlands e-mail: rvweeren@strw. leidenuniv.nl

2 Netherlands Institute for Radio Astronomy (ASTRON), Postbus 2, 7990 AA Dwingeloo, The Netherlands

3 Jacobs University Bremen, Campus Ring 1, 28759 Bremen, Germany

4 INAF/Istituto di Radioastronomia, via Gobetti 101, 40129 Bologna, Italy

5 Laboratoire Lagrange, UMR 7293, Université de Nice Sophia-Antipolis, CNRS, Observatoire de la Côte d'Azur, 06300 Nice, France
6 Department of Astrophysics/IMAPP, Radboud University Nijmegen, PO Box 9010, 6500 GL Nijmegen, The Netherlands

7 GEPI, Observatoire de Paris-Meudon, 5 place Jules Janssen, 92190 Meudon, France

8 Max Planck Institute for Astrophysics, Karl Schwarzschild Str. 1, 85741 Garching, Germany

9 Kapteyn Astronomical Institute, PO Box 800, 9700 AV Groningen, The Netherlands

10 Jodrell Bank Center for Astrophysics, School of Physics and Astronomy, The University of Manchester, Manchester M13 9PL, UK

11 Onsala Space Observatory, Dept. of Earth and Space Sciences, Chalmers University of Technology, 43992 Onsala, Sweden

12 National Radio Astronomy Observatory, 520 Edgemont Road, Charlottesville, VA 22903-2475, USA

13 Naval Research Laboratory, 4555 Overlook Avenue SW, Washington D. C. 20375, USA

14 Department of Physics \& Astronomy, The Open University, UK

15 Space Science Department, Rutherford Appleton Laboratory, Chilton, UK

16 Thüringer Landessternwarte, Sternwarte 5, 07778 Tautenburg, Germany

17 Astronomical Institute "Anton Pannekoek", University of Amsterdam, Postbus 94249, 1090 GE Amsterdam, The Netherlands

18 Argelander-Institut für Astronomie, University of Bonn, Auf dem Hügel 71, 53121 Bonn, Germany

19 Max-Planck-Institut für Radioastronomie, Auf dem Hügel 69, 53121 Bonn, Germany

20 School of Physics and Astronomy, University of Southampton, Southampton, SO17 1BJ, UK

21 Harvard-Smithsonian Center for Astrophysics, Garden Street 60, Cambridge, MA, 02138, USA

22 Institute for Astronomy, University of Edinburgh, Royal Observatory of Edinburgh, Blackford Hill, Edinburgh EH9 3HJ, UK

23 Mt Stromlo Obs., Research School of Astronomy and Astrophysics, Australian National University, Weston, A.C.T. 2611, Australia

24 Centre de Recherche Astrophysique de Lyon, Observatoire de Lyon, 9 Av. Charles André, 69561 Saint Genis Laval Cedex, France

25 Leibniz-Institut für Astrophysik Potsdam (AIP), An der Sternwarte 16, 14482 Potsdam, Germany

26 Laboratoire de Physique et Chimie de l'Environnement et de l'Espace 3A, Avenue de la Recherche Scientifique, 45071 Orléans Cedex 2, France 